\title{
GLOBAL EXISTENCE OF SOLUTIONS OF THE FREE BOUNDARY PROBLEM FOR THE EQUATIONS OF MAGNETOHYDRODYNAMIC COMPRESSIBLE FLUID
}

\author{
PIOTR KACPRZYK \\ Institute of Mathematics and Cryptology, Cybernetics Faculty \\ Military University of Technology \\ S. Kaliskiego 2, 00-908 Warszawa, Poland \\ E-mail: p.kacprzyk@imbo.wat.edu.pl
}

\begin{abstract}
Global existence of solutions for equations describing a motion of magnetohydrodynamic compresible fluid in a domain bounded by a free surface is proved. In the exterior domain we have an electromagnetic field which is generated by some currents located on a fixed boundary. We have proved that the domain occupied by the fluid remains close to the initial domain for all time.
\end{abstract}

1. Introduction. In this paper we prove the existence of global solution to equations describing a motion of magnetohydrodynamic compresible fluid in a domain $\Omega_{t} \subset \mathbb{R}^{3}$ bounded by a free surface $S_{t}$. In a domain $D_{t} \subset \mathbb{R}^{3}$ which is exterior to $\Omega_{t}$ we have a gas under a constant pressure $p_{0}$. Moreover in the domain $D_{t}$ we have an electromagnetic field generated by some currents located on a fixed boundary $B$ of $D_{t}$.

In the domain $\Omega_{t}$ the motion is described by the following problem

$$
\begin{array}{ll}
\varrho\left(v_{t}+v \cdot \nabla v\right)-\operatorname{div} \mathbb{T}(v, p)-\mu_{1} \stackrel{1}{H} \cdot \nabla \stackrel{1}{H}+\mu_{1} \nabla \frac{H^{2}}{2}=f & \text { in } \tilde{\Omega}^{T}, \\
\varrho_{t}+\operatorname{div}(\varrho v)=0 & \text { in } \tilde{\Omega}^{T}, \\
\mu_{1} \stackrel{1}{t}^{\prime}=-\operatorname{rot} \stackrel{1}{E} & \text { in } \tilde{\Omega}^{T}, \\
\stackrel{1}{H}=\sigma_{1}\left(\stackrel{1}{E}+\mu_{1} v \times \stackrel{1}{H}\right) & \text { in } \tilde{\Omega}^{T}, \\
\operatorname{div}\left(\mu_{1} \stackrel{1}{H}\right)=0 & \text { in } \tilde{\Omega}^{T},
\end{array}
$$

2000 Mathematics Subject Classification: 35A05, 35R35, 76N10.

Key words and phrases: free boundary, global existence, Sobolev spaces, magnetohydrodynamic compressible fluid.

Research supported by KBN grant no. 2PO3A00223.

The paper is in final form and no version of it will be published elsewhere. 
where $\tilde{\Omega}^{T}=\bigcup_{0 \leq t \leq T} \Omega_{t} \times\{t\}, v=v(x, t)$ is the velocity of the fluid, $p=p(\varrho)$ the pressure, $\varrho=\varrho(x, t)$ the density, $\stackrel{1}{H}=\stackrel{1}{H}(x, t)$ is the magnetic field, $f=f(x, t)$ the external force field per unit mass, $\mu_{1}$ the constant magnetic permeability, $\sigma_{1}$ the constant electric conductivity, $\stackrel{1}{E}=\stackrel{1}{E}(x, t)$ the electric field,

$$
\mathbb{T}(v, p)=\mathbb{D}(v)-p I
$$

is the stress tensor, where by

$$
\mathbb{D}(v)=\left\{\mu\left(\partial_{x_{i}} v_{j}+\partial_{x_{j}} v_{i}\right)+(\nu-\mu) \delta_{i j} \operatorname{div} v\right\}_{i, j=1,2,3}
$$

we denote the dilatation tensor, where $\nu, \mu$ are the viscosity coefficients of the fluid and $I$ the unit matrix.

In the domain $D_{t}$ occupied by dielectric (gas) we assume that there is no fluid motion inside $(v=0)$. Therefore we have only the electromagnetic field described by the following system

$$
\begin{aligned}
& \mu_{2} \stackrel{2}{H_{t}}=-\operatorname{rot} \stackrel{2}{E} \quad \text { in } \tilde{D}^{T} \text {, } \\
& \operatorname{rot} \stackrel{2}{H}=\sigma_{2} \stackrel{2}{E} \quad \text { in } \tilde{D}^{T} \text {, } \\
& \operatorname{div}\left(\mu_{2} \stackrel{2}{H}\right)=0 \quad \text { in } \tilde{D}^{T},
\end{aligned}
$$

where $\tilde{D}^{T}=\bigcup_{0 \leq t \leq T} D_{t} \times\{t\}$.

On $S_{t}=\partial \Omega_{t} \cap \partial D_{t}$ we assume the following transmission and boundary conditions

$$
\begin{array}{ll}
\mathbb{T}(v, p) n=\left(-p_{0} I-\mu_{1} \stackrel{1}{H} \otimes \stackrel{1}{H}+\mu_{1} \frac{H^{2}}{2} I\right) n & \text { on } \tilde{S}^{T} \\
\frac{1}{\sigma_{1}} \stackrel{1}{H}=\frac{1}{\sigma_{2}} \stackrel{2}{H} & \text { on } \tilde{S}^{T}, \\
\stackrel{1}{E} \cdot \tau_{\alpha}=\stackrel{2}{E} \cdot \tau_{\alpha}, \quad \alpha=1,2 & \text { on } \tilde{S}^{T}, \\
v \cdot n=-\frac{\phi_{t}}{|\nabla \phi|} & \text { on } \tilde{S}^{T},
\end{array}
$$

where $\tilde{S}^{T}=\bigcup_{0 \leq t \leq T} S_{t} \times\{t\}, n$ is the unit outward vector to $\Omega_{t}$ and normal to $S_{t}, \tau_{\alpha}$, $\alpha=1,2$ is the tangent vector to $S_{t}, \phi(x, t)=0$ describes $S_{t}$ at least locally.

Next we assume the boundary conditions on $B$

$$
\begin{aligned}
& \stackrel{2}{H}=H_{*} \quad \text { on } B, \\
& \stackrel{2}{E}=E_{*} \quad \text { on } B,
\end{aligned}
$$

where $H_{*}$ and $E_{*}$ are connected by conditions

$$
\begin{gathered}
\sigma_{2} E_{* n}=\frac{1}{A_{\tau_{1}} A_{\tau_{2}}}\left(\partial_{\tau_{1}}\left(H_{* \tau_{2}} A_{\tau_{2}}\right)-\partial_{\tau_{2}}\left(H_{* \tau_{1}} A_{\tau_{1}}\right)\right), \\
\mu_{2} \partial_{t} H_{* n}=\frac{1}{A_{\tau_{1}} A_{\tau_{2}}}\left(\partial_{\tau_{2}}\left(E_{* \tau_{1}} A_{\tau_{1}}\right)-\partial_{\tau_{1}}\left(E_{* \tau_{2}} A_{\tau_{2}}\right)\right),
\end{gathered}
$$




$$
\begin{aligned}
& -\partial_{t} \partial_{\tau_{1}}\left(H_{* \tau_{1}} A_{\tau_{2}} A_{n}\right)-\partial_{t} \partial_{\tau_{2}}\left(H_{* \tau_{2}} A_{\tau_{1}} A_{n}\right)=\partial_{\tau_{1}} \partial_{\tau_{2}}\left(E_{* n} A_{n}\right) \\
& \quad-\mu_{2} \partial_{\tau_{1}}\left(A_{\tau_{2}} A_{\tau_{3}} \partial_{t} H_{* \tau_{1}}\right)-\mu_{2} \partial_{\tau_{2}}\left(A_{\tau_{1}} A_{\tau_{3}} \partial_{t} H_{* \tau_{2}}\right)-\partial_{\tau_{2}} \partial_{\tau_{1}}\left(E_{* n} A_{n}\right),
\end{aligned}
$$

where $\left(\tau_{1}, \tau_{2}, n\right)$ are curvilinear coordinates and $A_{\tau_{1}}, A_{\tau_{2}}, A_{n}$ are the Lamé coefficients of transformation $\left(\tau_{1}, \tau_{2}, n\right) \rightarrow\left(x_{1}, x_{2}, x_{3}\right)$.

Finally we assume the initial conditions

$$
\begin{aligned}
& \Omega_{t_{\mid} t=0}=\Omega, \quad S_{t_{\mid t=0}}=S, \quad D_{t_{\mid t=0}}=D, \\
& \varrho_{\mid t=0}=\varrho_{0}, \quad v_{\mid t=0}=v_{0}, \quad \stackrel{1}{H}_{\mid t=0}=\stackrel{1}{H}_{0}, \quad \text { in } \Omega, \\
& \stackrel{2}{H}_{\mid t=0}=\stackrel{2}{H}_{0}, \quad \text { in } D .
\end{aligned}
$$

The aim of this paper is to prove the existence of a global-in-time solution of problem (1.1)-(1.7) which remains for all time close to a constant state. Consider the equation

$$
p(\varrho)=p_{0},
$$

where $\varrho \in \mathbb{R}_{+}, p \in C^{3}\left(\mathbb{R}_{+}\right), p^{\prime}(\varrho)>0$.

Then, we introduce the following definition of the constant state.

DeFinition 1.1. Let $f=0$. Then by a constant (equilibrium) state we mean a solution $(v, \varrho)$ of problem (1.1)-(1.7) such that $v=0, \varrho=\varrho_{e}, \Omega_{t}=\Omega_{e}$ for $t \geq 0$, where $\varrho_{e}$ is a solution of $(1.8)$ and $\left|\Omega_{e}\right|=\frac{M}{\varrho_{e}}\left(\left|\Omega_{e}\right|=v o l \Omega_{e}\right)$, where $M=\int_{\Omega_{t}} \varrho(x, t) d x=\int_{\Omega} \varrho_{0}(\xi) d \xi$.

The first global existence theorems for equations describing the motion of compressible fluids were proved by V. A. Solonnikov and A. Tani [10] and independently by W. Zajączkowski [20, 21]. Both [10] and [20,21] are concerned with the barotropic case, but in $[20,21]$ it is assumed that the pressure of the fluid has the form $p=a \varrho^{\gamma}$, where $a>0$ and $\gamma>1$ are constants. A global existence result for the more general form of pressure, i.e. $p=p(\varrho)$, has been obtained in [16]. Moreover, global existence theorems for viscous compressible heat-conducting fluids can be found in $[13,14,16]$.

To prove existence of solutions to the above problem we introduce the Lagrangian coordinates $\xi \in \Omega$. The Lagrangian coordinates connected with the velocity $v$ are the initial data for the Cauchy problem

$$
\frac{d x}{d t}=v(x, t), \quad x_{\mid t=0}=\xi \in \Omega .
$$

Therefore $x_{v}(\xi, t)=\xi+\int_{0}^{t} \bar{v}(\xi, \tau) d \tau$, where

$$
\bar{v}(\xi, t)=v\left(x_{v}(\xi, t), t\right) .
$$

To introduce the Lagrangian coordinates in $D_{t}$ we extend $v$ on $D_{t}$. Let us denote the extended function by $v^{\prime}$. Then we define $\xi \in D$, by the Cauchy data to the problem

$$
\frac{d x}{d t}=v^{\prime}(x, t), \quad x_{\mid t=0}=\xi \in D .
$$

Therefore $x_{v^{\prime}}(\xi, t)=\xi+\int_{0}^{t} \bar{v}^{\prime}(\xi, \tau) d \tau$, where $\bar{v}^{\prime}(\xi, t)=v^{\prime}\left(x_{v^{\prime}}(\xi, t), t\right)$. Then by $(1.5)$

$$
\begin{aligned}
& \Omega_{t}=\left\{x \in \mathbb{R}^{3}: x=x_{v}(\xi, t), \xi \in \Omega\right\}, \\
& S_{t}=\left\{x \in \mathbb{R}^{3}: x=x_{v}(\xi, t), \xi \in S\right\} .
\end{aligned}
$$


Since $S_{t}$ is determined at least locally by $\phi(x, t)=0, S$ is described by $\phi\left(x_{v}(\xi, t), t\right)_{\mid t=0}$ $=0$. Moreover, we have

$$
\bar{n}_{v}=n\left(x_{v}(\xi, t), t\right)=\left.\frac{\nabla_{x} \phi(x, t)}{\left|\nabla_{x} \phi(x, t)\right|}\right|_{x=x_{v}(\xi, t)} .
$$

Introduce the following notation:

$$
\begin{array}{ll}
\|u\|_{l, Q}=\|u\|_{H^{l}(Q)}, & Q \in\{\Omega, S, D, \Pi, B\}, \quad 0 \leq l \in \mathbb{Z}, \\
\|u\|_{k, p, q, Q^{T}}=\|u\|_{L_{q}\left(0, T, W_{p}^{k}(Q)\right)}, & Q \in\{\Omega, S, D, \Pi, B\}, \\
p, q \in[1,+\infty], \quad 0 \leq k \in \mathbb{Z}, &
\end{array}
$$

where $Q^{t}=Q \times(0, t)$,

$$
|u|_{p, Q}=\|u\|_{L_{p}(Q)}, \quad Q \in\{\Omega, S, D, \Pi, B\}, \quad p \in[1,+\infty] .
$$

2. Weak solution. Weak solutions to problem $(1.1)-(1.7)$ we formulate in Lagrangian coordinates.

Definition 2.1. By weak solutions for problem (1.1)-(1.7) we mean functions $\bar{v}, \bar{H}$ which satisfy the integral identities

$$
\begin{aligned}
& \int_{0}^{T} \int_{\Omega}\left(\varrho \bar{v}_{t} \bar{\varphi}+\mathbb{D}_{v}(\bar{v}) \mathbb{D}_{v}(\bar{\varphi})\right) I_{v} d \xi d t-\int_{0}^{T} \int_{\Omega}\left(\mu_{1} \stackrel{1}{\bar{H}} \nabla_{v} \stackrel{1}{\bar{H}} \bar{\varphi}-\mu_{1} \nabla_{v} \bar{H}^{2} \bar{\varphi}\right) I_{v} d \xi d t \\
& =\int_{0}^{T} \int_{\Omega} \bar{f} \bar{\varphi} I_{v} d \xi d t+\int_{0}^{T} \int_{S}\left(-\mu_{1} \bar{H} \otimes \frac{1}{\bar{H}}+\mu_{1} \frac{\bar{H}^{2}}{2} I\right) \bar{n}_{v} \bar{\varphi} I_{v} d \xi_{S} d t I_{v} d \xi_{s} d t \\
& \quad+\int_{0}^{T} \int_{\Omega} \nabla_{v} \bar{p} I_{v} d \xi d t-\int_{0}^{T} \int_{S}\left(\bar{p}-p_{0}\right) \bar{n}_{v} \bar{\varphi} I_{v} d \xi_{s} d t \\
& \int_{0}^{T} \int_{\Pi}\left(-\mu \bar{H}_{t} \bar{\psi}-\mu \bar{v} \nabla_{v} \bar{H} \bar{\psi}+\frac{1}{\sigma} \operatorname{rot}_{v} \bar{H} \operatorname{rot}_{v} \bar{\psi}\right) I_{v} d \xi d t \\
& \quad-\int_{0}^{T} \int_{\Omega} \mu_{1}\left(\bar{v} \times \frac{1}{H}\right) \operatorname{rot}_{v} \bar{\psi} I_{v} d \xi d t=\frac{1}{\sigma_{2}} \int_{0}^{T} \int_{B}\left(\bar{n}_{v} \times \bar{E}_{*}\right) \bar{\psi} I_{v} d \xi_{B} d t
\end{aligned}
$$

where $\varphi, \psi$ are sufficiently regular, $\bar{n}_{v}$ is the unit outward vector normal to $S$ or $B$. In $(2.1),(2.2)$ we use the notation $\bar{A}(\xi, t)=A\left(x_{v}(\xi, t), t\right)$,

$$
\begin{aligned}
& \bar{H}_{\mid \Omega}=\stackrel{1}{\bar{H}}, \bar{H}_{\mid D}=\stackrel{2}{\bar{H}}, \quad \sigma_{\mid \Omega}=\sigma_{1}, \quad \sigma_{\mid D}=\sigma_{2}, \\
& \Pi=\Omega \cup D, \quad \mu_{\mid \Omega}=\mu_{1}, \quad \mu_{\mid D}=\mu_{2},
\end{aligned}
$$

in $(2.2) v$ is the extension on $\Pi$,

$$
\begin{aligned}
\mathbb{D}_{v}(\bar{v}) & =\left\{\mu\left(\partial_{x_{i}} \xi_{k} \nabla_{\xi_{k}} \bar{v}_{j}+\partial_{x_{j}} \xi_{k} \nabla_{\xi_{k}} \bar{v}_{i}\right)+(\nu-\mu) \delta_{i j} \operatorname{div}_{v} \bar{v}\right\}_{i, j=1,2,3}, \\
\operatorname{rot}_{v} \bar{v} & =\nabla_{v} \times \bar{v}, \\
\nabla_{v} & =\partial_{x} \xi_{i} \nabla_{\xi_{i}}, \quad \operatorname{div}_{v} \bar{v}=\nabla_{v} \cdot \bar{v}=\partial_{x_{i}} \xi_{k} \nabla_{\xi_{k}} \bar{v}_{i}, \quad \partial_{\xi_{i}}=\nabla_{\xi_{i}} .
\end{aligned}
$$

Let $A$ be the Jacobi matrix of the transformation $x=x_{v}(\xi, t)$, then

$$
\operatorname{det} A=\exp \left(\int_{0}^{t} \operatorname{div}_{v} \bar{v} d \tau\right)=I_{v} \text { and if } \sup _{\xi \in \Omega} \sup _{t \in[0, T]}\left|\nabla_{\xi} \bar{v}\right|<\mu
$$


then $0<c_{1}(1-\mu t)^{3} \leq \operatorname{det}\left\{\partial_{\xi} x\right\} \leq c_{2}(1+\mu t)^{3}, t \in[0, T]$, where $c_{1}, c_{2}$ are constants and $T$ is sufficiently small. Moreover $x_{i \xi_{j}}=\delta_{i j}+\int_{0}^{t} \partial_{\xi_{j}} \bar{v}_{i}(\xi, \tau) d \tau$ and $\xi_{x}=x_{\xi}^{-1}$. Then we get

$$
\begin{aligned}
\sup _{\xi \in \Omega}\left|x_{\xi}\right| & \leq 1+\sup _{\xi \in \Omega} \int_{0}^{t}|\bar{v}(\xi, t)| d t \\
& \leq 1+c \int_{0}^{t}\|\bar{v}\|_{3, \Omega} d \tau \leq 1+c \sqrt{t} \sqrt{\int_{0}^{t}\|\bar{v}\|_{3, \Omega}^{2} d \tau} \leq 1+c \sqrt{t}\|\bar{v}\|_{3,2,2, \Omega^{t}} .
\end{aligned}
$$

Then $\sup _{x \in \Omega_{t}}\left|\xi_{x}\right| \leq \varphi(a)$, where $a=\sqrt{t}\|\bar{v}\|_{3,2,2, \Omega^{t}}$ and $\varphi$ is an increasing positive function.

To prove the existence of a solution to the above problem we introduce Lagrangian coordinates connected with given divergence-free function $u$. Moreover we linearize the nonlinear terms with $v$ in (1.1) writing them in the form $u \nabla v$ and $u \times \stackrel{1}{H}$. Then from (2.1), (2.2) we get

$$
\begin{aligned}
& \int_{0}^{T} \int_{\Omega}\left(\bar{\varrho} \bar{v}_{t} \bar{\varphi}+\mathbb{D}_{u}(\bar{v}) \mathbb{D}_{u}(\bar{\varphi})\right) I_{u} d \xi d t-\int_{0}^{T} \int_{\Omega}\left(\mu_{1} \stackrel{1}{\bar{H}^{\prime}} \nabla_{u}{\stackrel{1}{H^{\prime}}}^{\prime} \bar{\varphi}-\mu_{1} \nabla_{u}{\stackrel{1}{H^{\prime}}}^{2} \bar{\varphi}\right) I_{u} d \xi d t \\
& =\int_{0}^{T} \int_{\Omega} \bar{f} \bar{\varphi} I_{u} d \xi d t+\int_{0}^{T} \int_{S}\left(-\mu_{1} \stackrel{1}{\bar{H}} \otimes \bar{H}^{\frac{1}{H}}+\mu_{1} \frac{\bar{H}^{2}}{2} I\right) \bar{n}_{u} \bar{\varphi} I_{u} d \xi_{S} d t d \xi_{s} d t \\
& +\int_{0}^{T} \int_{\Omega} \nabla_{u} \bar{p} I_{u} d \xi d t-\int_{0}^{T} \int_{S}\left(\bar{p}-p_{0}\right) \bar{n}_{u} \bar{\varphi} I_{u} d \xi_{S} d t \\
& \int_{0}^{T} \int_{\Pi}\left(-\mu \bar{H}_{t} \bar{\psi}-\mu \bar{u} \nabla_{u} \bar{H} \bar{\psi}+\frac{1}{\sigma} \operatorname{rot}_{u} \bar{H} \operatorname{rot}_{u} \bar{\psi}\right) I_{u} d \xi d t+ \\
& -\int_{0}^{T} \int_{\Omega} \mu_{1}(\bar{u} \times \stackrel{1}{H}) \operatorname{rot}_{u} \bar{\psi} I_{u} d \xi d t=\frac{1}{\sigma_{2}} \int_{0}^{T} \int_{B}\left(\bar{n}_{u} \times \bar{E}_{*}\right) \bar{\psi} I_{u} d \xi_{B} d t
\end{aligned}
$$

where $\bar{u}, \overline{1}^{\prime}$ are given functions and moreover $\bar{\varrho}$ is such that

$$
0<\varphi_{*} \leq \bar{\varrho} \leq \varphi^{*}<\infty .
$$

Similarly as in [6] we prove

Theorem 2.1. Assume that $\bar{v}_{0} \in H^{2}(\Omega) ; \bar{v}_{t}(0) \in H^{1}(\Omega) ; \bar{v}_{t t}(0) \in L_{2}(\Omega) ; \bar{f}_{t} \in L_{2}(0, T$, $\left.H^{1}(\Omega)\right) ; \bar{f}_{t t} \in L_{2}\left(0, T, L_{2}(\Omega)\right) ; \bar{f} \in L_{2}\left(0, T, H^{2}(\Omega)\right) ; \bar{H}_{0} \in H^{2}(\Pi) ; \bar{H}_{t}(0) \in H^{1}(\Pi)$; $\bar{H}_{t t}(0) \in L_{2}(\Pi) ; \bar{E}_{*} \in L_{\infty}\left(0, T, H^{1}(B)\right) ; \bar{E}_{* t}, \bar{H}_{* t t} \in L_{2}\left(0, T, L_{2}(B)\right) ; \bar{H}_{* t} \in L_{2}(0, T$, $\left.H^{2}(B)\right) ; \bar{H}_{*} \in L_{2}\left(0, T, H^{3}(B)\right), S, B \in H^{5 / 2}$.

Then there exists $T^{*}>0$ such that for $T \leq T^{*}$ there exists a solution to problem (1.1)-(1.7) such that $\bar{v} \in L_{2}\left(0, T, H^{3}(\Omega)\right) \cap L_{\infty}\left(0, T, H^{1}(\Omega)\right) ; \bar{v}_{t} \in L_{\infty}\left(0, T, H^{1}(\Omega)\right) \cap$ $L_{2}\left(0, T, H^{2}(\Omega)\right) ; \bar{v}_{t t} \in L_{\infty}\left(0, T, L_{2}(\Omega)\right) \cap L_{2}\left(0, T, H^{1}(\Omega)\right) ; \bar{p}_{\sigma} \in L_{2}\left(0, T, H^{2}(\Omega)\right) ; \bar{p}_{t} \in$ $L_{2}\left(0, T, H^{1}(\Omega)\right) ; \bar{p}_{t t} \in L_{2}\left(0, T, L_{2}(\Omega)\right) ; \bar{H} \in L_{2}\left(0, T, H^{3}(\Pi)\right) \cap L_{\infty}\left(0, T, H^{1}(\Pi)\right) ; \bar{H}_{t} \in$ $L_{\infty}\left(0, T, H^{1}(\Pi)\right) \cap L_{2}\left(0, T, H^{2}(\Pi)\right) ; \bar{H}_{t t} \in L_{\infty}\left(0, T, L_{2}(\Pi)\right) \cap L_{2}\left(0, T, H^{1}(\Pi)\right)$. Similarly as in [5] we can prove that $\left(T^{*}\right)^{\gamma}(\varphi(0)+\beta) \leq b, \quad b>0$ sufficiently small, $\gamma>0$ some constant and 


$$
\begin{gathered}
\beta=\left\|\bar{E}_{*}\right\|_{0,2,2, B^{t}}^{2}+\left\|\bar{E}_{* t}\right\|_{0,2,2, B^{t}}^{2}+\left\|\bar{H}_{*}\right\|_{3,2,2, B^{t}}^{2}+\left\|\bar{H}_{* t}\right\|_{2,2,2, B^{t}}^{2}+\left\|\bar{H}_{* t t}\right\|_{0,2,2, B^{t}}^{2} \\
+\left\|\bar{f}_{t}\right\|_{0,2,2, \Omega^{t}}^{2}+\|\bar{f}\|_{1,2,2, \Omega^{t}}^{2}, \\
\varphi(0)=\sum_{i+k \leq 2}\left(\left\|\partial_{t}^{i} \bar{v}(0)\right\|_{k, \Omega}^{2}+\left\|\partial_{t}^{i} \bar{H}(0)\right\|_{k, \Pi}^{2}\right) .
\end{gathered}
$$

Moreover if $\varphi(0), \beta$ are sufficiently small then we get

$$
\begin{aligned}
& \left\|\bar{v}_{t}\right\|_{1,2, \infty, \Omega^{T}}^{2}+\|\bar{v}\|_{1,2, \infty, \Omega^{T}}^{2}+\|\bar{v}\|_{3,2,2, \Omega^{T}}^{2}+\left\|\bar{v}_{t}\right\|_{2,2,2, \Omega^{T}}^{2}+\left\|\bar{v}_{t t}\right\|_{1,2,2, \Omega^{T}}^{2} \\
& +\left\|\bar{p}_{\sigma}\right\|_{2,2,2, \Omega^{T}}^{2}+\left\|\bar{p}_{t t}\right\|_{0,2,2, \Omega^{T}}^{2}+\left\|\bar{p}_{t}\right\|_{1,2,2, \Omega^{T}}^{2}+\left\|\bar{H}_{t}\right\|_{1,2, \infty, \Pi^{T}}^{2}+\|\bar{H}\|_{1,2, \infty, \Pi^{T}}^{2} \\
& +\|\bar{H}\|_{3,2,2, \Pi^{T}}^{2}+\left\|\bar{v}_{t t}\right\|_{0,2, \infty, \Omega^{T}}^{2}+\left\|\bar{H}_{t}\right\|_{2,2,2, \Pi^{T}}^{2}+\left\|\bar{H}_{t t}\right\|_{1,2,2, \Pi^{T}}^{2}+\left\|\bar{H}_{t t}\right\|_{0,2, \infty, \Pi^{T}}^{2} \\
& \leq c(\varphi(0)+\beta) .
\end{aligned}
$$

First, in Section 3, we derive differential inequality (3.62) which makes possible an extension of the local solution of $(1.1)-(1.7)$ step by step from interval $[0, T]$ to $[0, \infty)$. In Section 4 we show the Main Theorem.

Main Theorem. Assume that $f=\int_{\Omega} v_{0} d x=\int_{\Omega} \varrho_{0} v_{0} \cdot \varphi_{i} d x=0, i=1,2,3$, where $\varphi_{i}, i=1,2,3$ are defined in Lemma 5.1, $H_{*} \in H^{3}(B), H_{* t} \in H^{2}(B), H_{* t t} \in H^{1}(B)$, $S_{t}, B \in H^{\frac{5}{2}},(v(0), \varrho(0), H(0)) \in \mathcal{N}(0), \varphi(0) \leq \varepsilon_{1}$ where $\varepsilon_{1}$ is sufficiently small. Assume also that $\alpha(t) \leq e^{-\mu t}$, where $\mu$ is sufficiently large and $\alpha(t)$ is defined in Lemma 4.2. Then there exists a global solution of (1.1)-(1.7) such that $\left(v(t), \varrho_{\sigma}(t), H(t)\right) \in \mathcal{M}(t)$, $t \in \mathbb{R}_{+}$, where $\mathcal{N}(0)$ and $\mathcal{M}(t)$ are defined in Section 4 .

3. Differential inequality. In this section we obtain a special differential inequality which enables us to prove the global existence. In order to show the differential inequality we consider the motion near the constant state. Let

$$
\begin{aligned}
& p_{\sigma}=p-p_{0}, \quad \varrho_{\sigma}=\varrho-\varrho_{e}, \quad \stackrel{2}{H}_{\mid B}=0, \quad f=0, \\
& \int_{\Omega} \varrho(0) v_{0} d x=0, \quad \int_{\Omega} \varrho(0) v_{0} \cdot \varphi_{i} d x=0, \quad i=1,2,3,
\end{aligned}
$$

where $\varrho_{e}$ is introduced in Definition 1.1 and $\varphi_{i}, i=1,2,3$ are defined in Lemma 5.1.

REMARK 3.1. Integrating $(1.1)_{1}$ over $\Omega_{t}$ we get

$$
\frac{d}{d t} \int_{\Omega_{t}} \varrho v d x-\int_{\Omega_{t}} \operatorname{div} \mathbb{T}\left(v, p_{\sigma}\right) d x+\mu_{1} \int_{\Omega_{t}}\left(-\operatorname{div}(\stackrel{1}{H} \otimes \stackrel{1}{H})+\nabla \frac{1}{H^{2}}\right) d x=\int_{\Omega_{t}} f d x .
$$

Then from (3.1) we get

$$
\frac{d}{d t} \int_{\Omega_{t}} \varrho v d x+\int_{S_{t}}\left(\mu_{1} \stackrel{1}{H} \otimes \stackrel{1}{H}-\mu_{1} \frac{H^{2}}{2} I\right) n d x_{S_{t}}+\mu_{1} \int_{\Omega_{t}}\left(-\operatorname{div}(\stackrel{1}{H} \otimes \stackrel{1}{H})+\nabla \frac{H^{2}}{2}\right) d x=0 .
$$

Integrating the last equality by parts we get

$$
\int_{\Omega_{t}} \varrho v d x=\int_{\Omega} \varrho(0) v_{0} d x=0
$$


REMARK 3.2. Let $\varphi_{i}, i=1,2,3$ are defined in Lemma 4.1. Multiplying $(1.1)_{1}$ by $\varphi_{i}$, $i=1,2,3$ and integrating over $\Omega_{t}$ we get

$$
\begin{aligned}
& \begin{array}{l}
\frac{d}{d t} \int_{\Omega_{t}} \varrho v \cdot \varphi_{i} d x-\int_{\Omega_{t}} \operatorname{div}\left(\mathbb{T}\left(v, p_{\sigma}\right) \varphi_{i}\right) d x+\mu_{1} \int_{\Omega_{t}}\left(-\operatorname{div}\left(\stackrel{1}{H} \otimes \stackrel{1}{H} \varphi_{i}\right)+\nabla \frac{H^{2}}{2} \varphi_{i}\right) d x \\
=\int_{\Omega_{t}} f d x .
\end{array} \\
& \text { Then from (3.1) we get }
\end{aligned}
$$

$$
\begin{aligned}
& \frac{d}{d t} \int_{\Omega_{t}} \varrho v \cdot \varphi_{i} d x+\int_{S_{t}}\left(\mu_{1} \stackrel{1}{H} \otimes \stackrel{1}{H}-\mu_{1} \frac{H^{2}}{2}\right) \varphi_{i} \cdot n d x_{S_{t}} \\
& \quad+\mu_{1} \int_{\Omega_{t}}\left(-\operatorname{div}(\stackrel{1}{H} \otimes \stackrel{1}{H}) \cdot \varphi_{i}+\nabla \frac{H^{2}}{2} \cdot \varphi_{i}\right) d x=0 .
\end{aligned}
$$

Integrating last equality by parts we get

$$
\int_{\Omega_{t}} \varrho v \cdot \varphi_{i} d x=\int_{\Omega} \varrho(0) v_{0} \cdot \varphi_{i} d x=0, \quad i=1,2,3 .
$$

Then we get the problem

$$
\begin{array}{ll}
\varrho\left[v_{t}+(v \cdot \nabla) v\right]-\operatorname{div} \mathbb{T}\left(v, p_{\sigma}\right)-\mu_{1} \stackrel{1}{H} \cdot \nabla \stackrel{1}{H}+\mu_{1} \nabla \frac{H^{2}}{2}=0 & \text { in } \Omega_{t}, \quad t \in[0, T], \\
\varrho_{\sigma t}+\operatorname{div}(\varrho v)=0 & \text { in } \Omega_{t}, \quad t \in[0, T], \\
\mathbb{T}\left(v, p_{\sigma}\right) \bar{n}=\left(-\mu_{1} \stackrel{1}{H} \otimes \stackrel{1}{H}+\mu_{1} \frac{H^{2}}{2} I\right) n & \text { on } S_{t}, \quad t \in[0, T], \\
\left.\varrho_{\sigma}\right|_{t=0}=\varrho_{\sigma 0}=\varrho_{0}-\varrho_{e},\left.v\right|_{t=0}=v_{0}, & \text { in } \Omega .
\end{array}
$$

In the sequel we shall use the following Taylor formula for $p_{\sigma}$

$$
p_{\sigma}=\left(\varrho-\varrho_{e}\right) \int_{0}^{1} p^{\prime}\left(\varrho_{e}+s\left(\varrho-\varrho_{e}\right)\right) d s=p_{1} \varrho_{\sigma},
$$

where the function $p_{1}$ is positive.

LEMMA 3.1. Let $v, \varrho_{\sigma}$ be a sufficiently smooth solution of (3.2). Then

$$
\frac{1}{2} \frac{d}{d t} \int_{\Omega_{T}}\left(\varrho v^{2}+\frac{p_{1}}{\varrho} \varrho_{\sigma}^{2}\right) d x+c_{0}\|v\|_{1, \Omega_{t}}^{2} \leq c X_{1}^{2}\left(1+X_{1}\right),
$$

where $X_{1}=\|v\|_{2, \Omega_{t}}^{2}+\left\|\varrho_{\sigma}\right\|_{2, \Omega_{t}}^{2}+\|\stackrel{1}{H}\|_{1, \Omega_{t}}^{2}$.

Proof. Multiplying $(3.2)_{1}$ by $v$, integrating over $\Omega_{t}$ and using continuity equation $(3.2)_{2}$, boundary condition $(3.2)_{3}$ and (3.3) we obtain

$$
\begin{aligned}
& \frac{1}{2} \frac{d}{d t} \int_{\Omega_{t}} \varrho v^{2} d x+\frac{\mu}{2} \mathbb{E}_{\Omega_{t}}(v)+(\nu-\mu)\|\operatorname{div} v\|_{0, \Omega_{t}}^{2}-\int_{\Omega_{t}} p_{1} \varrho_{\sigma} \operatorname{div} v d x \\
& -\mu_{1} \int_{\Omega_{t}} \stackrel{1}{H} \cdot \nabla \stackrel{1}{H} v d x+\mu_{1} \int_{\Omega_{t}} \nabla \frac{1}{2} v d x-\mu_{1} \int_{S_{t}}\left(-\stackrel{1}{H} \otimes \stackrel{1}{H}+\frac{H^{2}}{2} I\right) d x_{S}=0,
\end{aligned}
$$

where $\mathbb{E}_{\Omega_{t}}(v)=\int_{\Omega_{t}} \sum_{i, j=1}^{3}\left(v_{i x_{j}}+v_{j x_{i}}\right)^{2} d x$. 
In [21] it is proved that

$$
\frac{\mu}{2} \mathbb{E}_{\Omega_{t}}(v)+(\nu-\mu)\|\operatorname{div} v\|_{0, \Omega_{t}}^{2} \geq\left(\nu-\frac{1}{3} \mu\right) \mathbb{E}_{\Omega_{t}}(v) \quad \text { for } \quad \nu \geq \frac{1}{3} \mu .
$$

Next, by the continuity equation $(3.2)_{2}$ we have

$$
-\int_{\Omega_{t}} p_{1} \varrho_{\sigma} \operatorname{div} v d x=\frac{1}{2} \frac{d}{d t} \int_{\Omega_{t}} \frac{p_{1} \varrho_{\sigma}^{2}}{\varrho} d x+J
$$

where

$$
|J| \leq \varepsilon\left(\left\|\varrho_{\sigma t}\right\|_{0, \Omega_{t}}^{2}+\|v\|_{1, \Omega_{t}}^{2}\right)+c X_{1}^{2}\left(1+X_{1}\right) .
$$

By continuity equation $(3.2)_{2}$

$$
\left\|\varrho_{\sigma t}\right\|_{0, \Omega_{t}}^{2} \leq c\|v\|_{1, \Omega_{t}}^{2}+c\|v\|_{1, \Omega_{t}}^{2}\left\|\varrho_{\sigma}\right\|_{2, \Omega_{t}}^{2},
$$

and taking into account (3.5)-(3.8) we get estimate (3.4).

Next, we have

LEMMA 3.2. Let $v, \varrho_{\sigma}$ be a sufficiently smooth solution of (3.2). Then

$$
\frac{1}{2} \frac{d}{d t} \int_{\Omega_{t}}\left(\varrho v_{t}^{2}+\frac{p_{\varrho \sigma}}{\varrho} \varrho_{\sigma t}^{2}\right) d x+c_{0}\left(\left\|v_{t}\right\|_{1, \Omega_{t}}^{2}+\left\|\varrho_{\sigma t}\right\|_{0, \Omega_{t}}^{2}\right) \leq c\|v\|_{1, \Omega_{t}}^{2}+c Y_{1}^{2}\left(1+X_{2}\right),
$$

where

$$
\begin{gathered}
X_{2}=\sum_{i+k \leq 2}\left(\left\|\partial_{t}^{i} v\right\|_{k, \Omega_{t}}^{2}+\left\|\partial_{t}^{i} \varrho_{\sigma}\right\|_{k, \Omega_{t}}^{2}\right)+\int_{0}^{t}\|v\|_{3, \Omega_{\tau}}^{2} d \tau+\|\stackrel{1}{H}\|_{t, \Omega_{t}}^{2}+\|\stackrel{1}{H}\|_{1, \Omega_{t}}^{2}+\|v\|_{1, \Omega_{t}}^{2}, \\
Y_{1}=X_{2}-\int_{0}^{t}\|v\|_{3, \Omega_{\tau}}^{2} d \tau
\end{gathered}
$$

Proof. Differentiating $(3.2)_{1}$ with respect to $t$, multiplying by $v_{t}$ and integrating over $\Omega_{t}$ yields

$$
\begin{aligned}
\frac{1}{2} \frac{d}{d t} \int_{\Omega_{t}} \varrho v_{t}^{2} d x+\frac{\mu}{2} E_{\Omega_{t}}\left(v_{t}\right)+(\nu-\mu)\left\|\operatorname{div} v_{t}\right\|_{0, \Omega_{t}}^{2}-\int_{\Omega_{t}} p_{\sigma p} \varrho_{\sigma t} \operatorname{div} v_{t} d x & \leq c Y_{1}^{2}\left(1+X_{2}\right),
\end{aligned}
$$

where we have used boundary condition $(3.2)_{3}$.

By Lemma 5.2 and (*),

$$
\left\|v_{t}\right\|_{1, \Omega_{t}}^{2} \leq c\left[\mathbb{E}_{\Omega_{t}}\left(v_{t}\right)+Y_{1}^{2}\left(1+Y_{1}\right)\right]
$$

Finally, using continuity equation $(3.2)_{2}$ we get

$$
-\int_{\Omega_{t}} p_{\sigma \varrho} \varrho_{\sigma t} \operatorname{div} v_{t} d x=\frac{1}{2} \frac{d}{d t} \int_{\Omega_{t}} \frac{p_{\sigma \varrho}}{\varrho} \varrho_{\sigma t}^{2} d x+J
$$

where

$$
|J| \leq \varepsilon\left(\left\|v_{t}\right\|_{1, \Omega_{t}}^{2}+\left\|\varrho_{\sigma t}\right\|_{0, \Omega_{t}}^{2}\right)+c Y_{1}^{2}\left(1+Y_{1}\right) .
$$

In view of inequalities (3.12)-(3.15) and (3.8) we obtain (3.9).

Lemmas 3.1 and 3.2 yield 
LEMMA 3.3. Let $v, \varrho_{\sigma}$ be a sufficiently smooth solution of (3.2). Then

$$
\begin{array}{r}
\frac{1}{2} \frac{d}{d t} \int_{\Omega_{t}}\left[\varrho\left(v^{2}+v_{t}^{2}\right)+\frac{p_{1}}{\varrho} \varrho_{\sigma}^{2}+\frac{p_{\sigma \varrho}}{\varrho} \varrho_{\sigma t}^{2}\right] d x+c_{0}\left(\|v\|_{1, \Omega_{t}}^{2}+\left\|v_{t}\right\|_{1, \Omega_{t}}^{2}+\left\|\varrho_{\sigma t}\right\|_{0, \Omega_{t}}^{2}\right) \\
\leq c Y_{1}^{2}\left(1+X_{2}\right),
\end{array}
$$

where $X_{2}$ and $Y_{1}$ are given by (3.10) and (3.11), respectively.

Next, we obtain

LEMMA 3.4. Let $v, \varrho_{\sigma}$ be a sufficiently smooth solution of (3.2). Then

$$
\begin{aligned}
\frac{1}{2} \frac{d}{d t} \int_{\Omega_{t}}\left(\varrho v_{t t}^{2}+\frac{p_{\sigma \varrho}}{\varrho} \varrho_{\sigma t t}^{2}\right) d x+c_{0} & \left(\left\|v_{t t}\right\|_{1, \Omega_{t}}^{2}+\left\|\varrho_{\sigma t t}\right\|_{0, \Omega_{t}}^{2}\right) \\
& \leq c\left(\|v\|_{1, \Omega_{t}}^{2}+\left\|v_{t}\right\|_{1, \Omega_{t}}^{2}\right)+c X_{2} Y_{2}\left(1+X_{2}^{2}\right)
\end{aligned}
$$

where $X_{2}$ is given by (3.10) and

$$
\begin{aligned}
Y_{2}= & \sum_{\substack{i+k \leq 3 \\
i \leq 2}}\left\|\partial_{t}^{i} v\right\|_{k, \Omega_{t}}^{2}+\left\|\varrho_{\sigma}\right\|_{2, \Omega_{t}}^{2}+\left\|\varrho_{\sigma t}\right\|_{2, \Omega_{t}}^{2}+\left\|\varrho_{\sigma t t}\right\|_{1, \Omega_{t}}^{2}+\left\|\stackrel{1}{H}{ }_{t t}\right\|_{1, \Omega_{t}}^{2}+\|\stackrel{1}{H}\|_{2, \Omega_{t}}^{2} \\
& +\left\|\stackrel{1}{H}_{t}\right\|_{1, \Omega_{t}}^{2} .
\end{aligned}
$$

The above lemma can be proved in the same way as Lemmas 3.1 and 3.2. To estimate $\mathbb{E}_{\Omega_{t}}\left(v_{t t}\right)$ we use here Lemma 5.3.

In order to obtain estimates for derivatives with respect to $x$ we rewrite problem (3.2) in the Lagrangian coordinates. We have

$$
\begin{aligned}
& \bar{\varrho} \bar{v}_{t}-\nabla_{v} \mathbb{T}_{v}\left(\bar{v}, p_{\sigma}\right)-\mu_{1} \stackrel{\frac{1}{H}}{H} \cdot \nabla_{v} \stackrel{\frac{1}{H}}{H}+\mu_{1} \nabla_{v} \frac{\stackrel{1}{H}^{2}}{2}=0 \quad \text { in } \quad \Omega^{T} \equiv \Omega \times(0, T), \\
& \bar{\varrho}+\varrho \nabla_{v} \cdot \bar{v}=0 \quad \text { in } \Omega^{T} \text {, } \\
& \mathbb{T}_{v}\left(\bar{v}, p_{\sigma}\right) \bar{n}_{v}=\left(-\mu_{1} \stackrel{\frac{1}{H}}{\frac{1}{H}}+\mu_{1} \frac{\bar{H}^{2}}{2}\right) \bar{n}_{v} \quad \text { on } S^{T}=S \times(0, T), \\
& \left.\bar{v}\right|_{t=0}=v_{0},\left.\quad \bar{\varrho}\right|_{t=0}=\varrho_{\sigma 0} \quad \text { in } \Omega .
\end{aligned}
$$

Now, introduce a partition of unity $\left(\left\{\bar{\Omega}_{i}\right\},\left\{\zeta_{i}\right\}\right), \Omega=\bigcup_{i} \tilde{\Omega}_{i}$. Let $\tilde{\Omega}$ be one of the $\tilde{\Omega}_{i}, s$ and $\zeta(\xi)=\zeta_{i}(\xi)$ be the corresponding function. If $\tilde{\Omega}$ is an interior subdomain then let $\tilde{\omega}$ be a set such that $\tilde{\omega} \subset \tilde{\Omega}$ and $\zeta(\xi)=1$ for $\xi \in \tilde{\omega}$. Otherwise, we assume that $\overline{\tilde{\Omega}} \cap S \neq \emptyset$, $\overline{\tilde{\omega}} \cap S \neq \emptyset, \overline{\tilde{\omega}} \subset \overline{\tilde{\Omega}}$. Take any $\beta \in \overline{\tilde{\omega}} \cap S=\overline{\tilde{S}}$ and introduce local coordinates $\{y\}$ associated with $\{\xi\}$ by

$$
y_{k}=\alpha_{k l}\left(\xi_{l}-\beta_{l}\right), \quad \alpha_{3 k}=n_{k}(\beta), \quad k=1,2,3,
$$

where $\left\{\alpha_{k l}\right\}$ is a constant orthogonal matrix such that $\tilde{S}$ is determined by the equation $y_{3}=F\left(y_{1}, y_{2}\right), F \in H^{\frac{5}{2}}$ and

$$
\tilde{\Omega}=\left\{y:\left|y_{i}\right|<d, i=1,2, F\left(y^{\prime}\right)<y_{3}<F\left(y^{\prime}\right)+d, y^{\prime}=\left(y_{1}, y_{2}\right)\right\} .
$$


Next, we introduce $\bar{v}^{\prime}, \bar{\varrho}^{\prime}, \bar{\varrho}_{\sigma}$ by

$$
\begin{array}{ll}
\bar{v}_{i}^{\prime}(y)=\left.\alpha_{i j} \bar{v}_{j}(\xi)\right|_{\xi=\xi(y)}, & i=1,2,3, \\
\bar{\varrho}^{\prime}(y)=\left.\bar{\varrho}(\xi)\right|_{\xi=\xi(y)}, \quad \bar{\varrho}_{\sigma}^{\prime}(y)=\bar{\varrho}^{\prime}(y)-\varrho_{e},
\end{array}
$$

where $\xi=\xi(y)$ is the inverse transformation to (3.20).

Next, we introduce new variables by

$$
z_{i}=y_{i}, \quad i=1,2, \quad z_{3}=y_{3}-\tilde{F}(y), \quad y \in \tilde{\Omega},
$$

which will be denoted by $z=\Phi(y)$ (where $\tilde{F} \in H^{3}$ is an extension of $F$ ). Let

$$
\hat{\Omega}=\Phi(\tilde{\Omega})=\left\{z:\left|z_{i}\right|<d, i=1,2,0<z_{3}<d\right\} \quad \text { and } \quad \hat{S}=\Phi(\tilde{S}) .
$$

Define

$$
\hat{v}(z)=\left.\bar{v}^{\prime}(y)\right|_{y=\Phi^{-1}(z)}, \quad \hat{\varrho}(z)=\left.\bar{\varrho}^{\prime}(y)\right|_{y=\Phi^{-1}(z)}, \quad \hat{\varrho}_{\sigma}(z)=\hat{\varrho}(z)-\varrho_{e} .
$$

Set $\hat{\nabla}_{k}=\left.\xi_{l x_{k}} z_{i \xi_{l}} \nabla_{z_{i}}\right|_{\xi=\chi^{-1}(z)}$, where $\chi(\xi)=\Phi(\psi(\xi))$ and $y=\psi(\xi)$ is described by (3.20). We also introduce the following notation:

$$
\tilde{v}(\xi)=\bar{v}(\xi) \zeta(\xi), \quad \tilde{\varrho}(\xi)=\bar{\varrho}(\xi) \zeta(\xi), \quad \tilde{\varrho}_{\sigma}(\xi)=\bar{\varrho}_{\sigma}(\xi) \zeta(\xi),
$$

for $\xi \in \tilde{\Omega}, \tilde{\Omega} \cap S=\emptyset$ and

$$
\tilde{v}(z)=\hat{v}(z) \hat{\zeta}(z), \quad \tilde{\varrho}(z)=\hat{\varrho}(z) \hat{\zeta}(z), \quad \hat{\varrho}_{\sigma}(z)=\hat{\varrho}_{\sigma}(z) \hat{\zeta}(z),
$$

for $z \in \hat{\Omega}=\Phi(\tilde{\Omega}), \overline{\tilde{\Omega}} \cap S \neq \emptyset$, where $\hat{\zeta}(z)=\left.\zeta(\xi)\right|_{\xi=\chi^{-1}(z)}$.

Using the above notation we rewrite problem (3.19) in the following form in an interior subdomain

$$
\begin{aligned}
& \bar{\varrho} \tilde{v}_{i t}-\nabla_{v_{j}} T_{v i j}\left(\tilde{v}, \tilde{p}_{\sigma}\right)=-\nabla_{v_{j}} B_{v i j}(\bar{v}, \zeta)-T_{v i j}\left(\bar{v}, p_{\sigma}\right) \nabla_{v_{j}} \zeta+\mu_{1}\left(\tilde{\tilde{H}} \nabla_{v} \stackrel{1}{\bar{H}}_{i}-\left(\nabla_{v_{i}} \stackrel{1}{\bar{H}}\right) \stackrel{1}{\tilde{H}}\right) \\
& \quad \equiv k_{1}, \quad i=1,2,3, \\
& \tilde{\varrho}_{\sigma t}+\underline{\varrho} \nabla_{v} \cdot \tilde{v}=\bar{\varrho} \bar{v} \cdot \nabla_{v} \zeta \equiv k_{2},
\end{aligned}
$$

where $\tilde{p}_{\sigma}=p_{\sigma} \zeta, \mathbb{B}_{v}(\bar{v}, \zeta)=\left\{B_{v}^{i j}(\bar{v}, \zeta)\right\}_{i, j=1,2,3}=\left\{\mu\left(\bar{v}_{i} \nabla_{v} \zeta+\bar{v}_{j} \nabla_{v} \zeta\right)+\right.$ $\left.(\nu-\mu) \delta_{i j} \bar{v} \nabla_{v} \zeta\right\}_{i, j=1,2,3}, \mathbb{T}_{v}\left(\bar{v}, p_{\sigma}\right)=\mathbb{D}_{v}(\bar{v})-I p_{\sigma}=\left\{T_{v i j}\left(\bar{v}, p_{\sigma}\right)\right\}_{i, j=1,2,3}$ and $\nabla_{v_{j}}=$ $\xi_{k x_{j}} \partial_{\xi_{k}}$.

In boundary subdomains we have

$$
\begin{aligned}
& \hat{\varrho} \tilde{v}_{i t}-\hat{\nabla}_{j} \hat{T}_{i j}\left(\tilde{v}, \tilde{p}_{\sigma}\right)=\hat{\nabla}_{j} \hat{B}_{i j}(\hat{v}, \hat{\zeta})-T_{v i j}\left(\hat{v}, \hat{p}_{\sigma}\right) \hat{\nabla_{j}} \hat{\zeta}+\mu_{1}\left(\tilde{H} \hat{\nabla} \hat{H}_{i}-\left(\hat{\nabla_{i}} \stackrel{1}{\hat{H}}\right) \stackrel{1}{\tilde{H}}\right) \\
& \quad \equiv k_{3 i}, \quad i=1,2,3, \\
& \tilde{\varrho}_{\sigma t}+\hat{\varrho} \hat{\nabla} \cdot \tilde{v}=\hat{\eta} \hat{v} \cdot \hat{\nabla} \hat{\zeta} \equiv k_{4}, \\
& \hat{\mathbb{T}}\left(\tilde{v}, \tilde{p}_{\sigma}\right) \hat{n}=k_{5},
\end{aligned}
$$

where $k_{5}=\hat{B}_{i j}(\hat{v}, \hat{\zeta}) \hat{n}+\left(-\mu_{1} \hat{H} \otimes \frac{1}{\hat{H}}+\mu_{1} \frac{\hat{H}^{2}}{2} I\right) \hat{n}, \hat{\nabla}=\left(\hat{\nabla}_{j}\right)_{j=1,2,3}$ and $\hat{\mathbb{T}}$ and $\hat{\mathbb{B}}$ indicate that the operator $\nabla_{v}$ is replaced by $\hat{\nabla}$.

In Lemmas 3.5-3.7 below we denote $z_{1}, z_{2}$ by $\tau$, i.e. $\tau=\left(z_{1}, z_{2}\right)$ and $z_{3}$ by $n$. 
LEMMA 3.5. Let $v, \varrho_{\sigma}$ be a sufficiently smooth solution of (3.2). Then

$$
\begin{aligned}
& \frac{1}{2} \frac{d}{d t} \int_{\Omega_{t}}\left(\varrho v_{x}^{2}+\frac{p_{\sigma \varrho}}{\varrho} \varrho_{\sigma x}^{2}\right) d x+c_{0}\left(\|v\|_{2, \Omega_{t}}^{2}+\left\|\varrho_{\sigma x}\right\|_{0, \Omega_{t}}^{2}\right) \\
& \quad \leq c\left(\|v\|_{1, \Omega_{t}}^{2}+\left\|v_{t}\right\|_{1, \Omega_{t}}^{2}+\left\|\varrho_{\sigma t}\right\|_{0, \Omega_{t}}^{2}+\left\|p_{\sigma}\right\|_{0, \Omega_{t}}^{2}\right)+c X_{2}^{2}\left(1+X_{2}\right),
\end{aligned}
$$

where $X_{2}$ is given by (3.10), $v_{x}^{2}=\sum_{i, j=1}^{3} v_{i x_{j}}^{2}, \varrho_{\sigma x}^{2}=\sum_{i=1}^{3} \varrho_{\sigma x_{i}}^{2}$.

Proof. First, we consider the following elliptic problem

$$
\begin{array}{ll}
\mu \nabla_{v}^{2} \bar{v}+\nu \nabla_{v} \nabla_{v} \cdot \bar{v}-p_{\sigma \varrho} \nabla_{v} \bar{\varrho}=\bar{\varrho}_{t}+\mu_{1} \frac{1}{\bar{H}} \cdot \nabla_{v} \frac{1}{\bar{H}}-\mu_{1} \nabla_{v} \frac{\bar{H}^{2}}{2} & \text { in } \Omega, \\
\operatorname{div}_{v} \bar{v}=\operatorname{div}_{v} \bar{v} & \text { in } \Omega, \\
\mathbb{T}_{v}\left(\bar{v}, p_{\sigma}\right) \bar{n}_{v}=\left(-\mu_{1} \frac{1}{\bar{H}} \otimes \frac{1}{\bar{H}}+\mu_{1} \frac{\bar{H}^{2}}{2} I\right) \bar{n}_{v} & \text { on } S .
\end{array}
$$

Since the complementary condition to (3.24) is satisfied we can apply to problem (3.24) the Agmon-Douglis-Nirenberg theory (see [1]). Thus, we get

$$
\begin{aligned}
& \|\bar{v}\|_{2, \Omega}^{2}+\left\|\bar{\varrho}_{\sigma}\right\|_{1, \Omega}^{2} \leq c\left(\left\|\bar{\varrho} \bar{v}_{t}\right\|_{0, \Omega}^{2}+\left\|\operatorname{div}_{v} \bar{v}\right\|_{1, \Omega}^{2}+\|\bar{H}\|_{1, \Omega}^{2}\right) \\
& \quad \leq c\left(\left\|\bar{v}_{t}\right\|_{0, \Omega}^{2}+\|\operatorname{div} \bar{v}\|_{1, \Omega}^{2}+c X_{2}^{2}(\Omega)\left(1+X_{2}(\Omega)\right)\right)+\|\bar{v}\|_{0, \Omega}^{2}
\end{aligned}
$$

where we have used that $\left\|\operatorname{div}_{u} \bar{v}-\operatorname{div} \bar{v}\right\|_{1, \Omega}^{2} \leq \varepsilon\|\bar{v}\|_{2, \Omega}^{2},(\varepsilon>0$ is sufficiently small $)$ and

$$
X_{2}(\Omega)=\sum_{i+k \leq 2}\left(\left\|\partial_{t}^{i} \bar{v}\right\|_{k, \Omega}^{2}+\left\|\partial_{t}^{i} \bar{\varrho}_{\sigma}\right\|_{k, \Omega}^{2}\right)+\int_{0}^{t}\|\bar{v}\|_{3, \Omega}^{2} d \tau+\|\stackrel{1}{\bar{H}}\|_{2, \Omega}^{2} .
$$

In view of (3.25) we see that in order to obtain inequality (3.23) it remains to get appropriate estimates for $\|\operatorname{div} \bar{v}\|_{1, \Omega}^{2}$ and for $\frac{1}{2} \frac{d}{d t} \int_{\Omega_{t}}\left(\varrho v_{x}^{2}+\frac{p_{\sigma \varrho}}{\varrho} \varrho_{\sigma x}^{2}\right) d x$. To do this we first consider boundary subdomains. Differentiate $(3.24)_{1}$ with respect to $\tau$, multiply the result by $\tilde{v}_{\tau} J(J$ is the Jacobian of the transformation $x=x(z))$ and integrate over $\hat{\Omega}$. Hence using the Korn inequality and equation $(3.22)_{2}$ we obtain

$$
\begin{aligned}
& \frac{1}{2} \frac{d}{d t} \int_{\hat{\Omega}} \hat{\varrho} \tilde{v}_{\tau}^{2} J d z+c_{0}\left\|\tilde{v}_{\tau}\right\|_{1, \hat{\Omega}}^{2}-\int_{\hat{S}}\left(\hat{\mathbb{T}}\left(\tilde{v}, \tilde{p}_{\sigma}\right) \hat{n}\right)_{\tau} \tilde{v}_{\tau} J d z \\
& \quad-\int_{\hat{\Omega}} \tilde{p}_{\sigma \tau} \nabla \cdot \tilde{v}_{\tau} J d z \leq \varepsilon\left(\left\|\hat{\varrho}_{\sigma}\right\|_{0, \hat{\Omega}}^{2}+\left\|\tilde{v}_{\tau}\right\|_{1, \hat{\Omega}}^{2}\right) \\
& \quad+c\left(\|\hat{v}\|_{1, \hat{\Omega}}^{2}+\left\|p_{\sigma}\right\|_{0, \hat{\Omega}}^{2}\right)+c X_{2}^{2}(\hat{\Omega})\left(1+X_{2}(\hat{\Omega})\right),
\end{aligned}
$$

where

$$
\begin{aligned}
X_{2}(\hat{\Omega}) & =\sum_{i+k \leq 2}\left(\left\|\partial_{t}^{i} \hat{v}\right\|_{k, \hat{\Omega}}^{2}+\left\|\partial_{t}^{i} \hat{\varrho}_{\sigma}\right\|_{k, \hat{\Omega}}^{2}\right)+\int_{0}^{t}\|\hat{v}\|_{3, \hat{\Omega}}^{2} d \tau+\|\hat{H}\|_{2, \hat{\Omega}}^{2}, \\
\tilde{v}_{\tau}^{2} & =\sum_{i=1}^{3} \sum_{j=1}^{2} \tilde{v}_{i z_{j}}^{2} .
\end{aligned}
$$


Using boundary condition $(3.24)_{3}$ we have

$$
\begin{aligned}
-\int_{\hat{S}}\left(\hat{\mathbb{T}}\left(\tilde{v}, \tilde{p}_{\sigma}\right) \hat{n}\right)_{\tau} \tilde{v}_{\tau} J d \tau & =-\int_{\hat{S}}\left(\hat{B}_{i j}(\tilde{v}, \hat{\zeta}) \hat{n}_{j}\right)_{\tau} \tilde{v}_{\tau} J d \tau \\
& =\int_{\hat{S}} \partial_{\tau}^{1 / 2}\left(\hat{B}_{i j}(\hat{v}, \hat{\zeta}) \hat{n}_{j}\right) \partial_{\tau}^{1 / 2}\left(\tilde{v}_{i \tau} J\right) d \tau \\
& \leq \varepsilon\left\|\tilde{v}_{\tau}\right\|_{1, \hat{\Omega}}^{2}+\|\hat{v}\|_{1, \hat{\Omega}}^{2}+c X_{2}^{2}(\hat{\Omega})
\end{aligned}
$$

where to use derivative $\partial_{\tau}^{1 / 2}$ we have to apply the Fourier transform.

Next,

$$
-\int_{\hat{\Omega}} \tilde{p}_{\sigma \tau} \nabla_{v} \cdot \tilde{v}_{\tau} J d z=-\int_{\hat{\Omega}} p_{\sigma \varrho} \tilde{\varrho}_{\sigma \tau} \hat{\nabla} \cdot \tilde{v}_{\tau} J d z+J_{1},
$$

where $\left|J_{1}\right| \leq \varepsilon\left\|\tilde{v}_{\tau}\right\|_{1, \hat{\Omega}}^{2}+c\left\|p_{\sigma}\right\|_{0, \hat{\Omega}}^{2}$ and

$$
-\int_{\hat{\Omega}} p_{\sigma \hat{\varrho}} \hat{\varrho}_{\sigma \tau} \hat{\nabla} \cdot \tilde{v}_{\tau} J d z=\frac{1}{2} \frac{d}{d t} \int_{\hat{\Omega}} \frac{p_{\sigma} \hat{\varrho}}{\varrho} \tilde{\varrho}_{\sigma \tau}^{2} J d z+J_{2},
$$

where

$$
\left|J_{2}\right| \leq \varepsilon\left\|\tilde{\varrho}_{\sigma \tau}\right\|_{0, \hat{\Omega}}^{2}+c\|\hat{v}\|_{1, \hat{\Omega}}^{2}+c X_{2}^{2}(\hat{\Omega}) .
$$

Taking into account (3.27), (3.29)-(3.32) and assuming that $\varepsilon$ is sufficiently small we obtain

$$
\begin{aligned}
& \frac{1}{2} \frac{d}{d t} \int_{\hat{\Omega}}\left(\hat{\varrho} \tilde{v}_{\tau}+\frac{p_{\sigma \hat{\varrho}}}{\hat{\varrho}} \tilde{\varrho}_{\sigma \tau}^{2}\right) J d z+c_{0}\left\|\tilde{v}_{\tau}\right\|_{1, \hat{\Omega}}^{2} \\
& \quad \leq \varepsilon\left\|\hat{\varrho}_{\sigma \tau}\right\|_{0, \hat{\Omega}}^{2}+c\left(\|\hat{v}\|_{1, \hat{\Omega}}^{2}+\left\|p_{\sigma}\right\|_{0, \hat{\Omega}}^{2}\right)+c X_{2}^{2}(\hat{\Omega})\left(1+X_{2}(\hat{\Omega})\right) .
\end{aligned}
$$

Now, applying the operator $(\mu+\nu) \nabla_{z_{i}}$ to $(3.22)_{2}$, dividing the result by $\hat{\varrho}$, adding to $(3.22)_{1}$ and multiplying both sides of the result by $p_{\sigma \varrho}$ gives

$$
\begin{aligned}
& \frac{(\mu+\nu)}{\varrho} p_{\sigma \varrho} \nabla_{z_{i}} \hat{\varrho}_{\sigma_{t}}+p_{\sigma \hat{\varrho}}^{2} \nabla_{z_{i}} \hat{\varrho}_{\sigma}=p_{\sigma \varrho}^{2} \hat{\varrho}_{\sigma} \nabla_{z_{i}} \hat{\zeta}-p_{1} p_{\sigma \varrho} \hat{\varrho}_{\sigma} \nabla_{z_{i}} \hat{\zeta} \\
& \quad+p_{\sigma} k_{3 i}+\mu p_{\sigma \hat{\varrho}}\left(\hat{\nabla}^{2} \tilde{v}_{i}-\hat{\nabla}_{i} \hat{\nabla} \cdot \tilde{v}\right) \\
& \quad+(\mu+\nu) p_{\sigma \hat{\eta}}\left(\hat{\nabla}_{i}-\nabla_{z_{i}}\right) \hat{\nabla} \cdot \tilde{v}+\frac{(\mu+\nu)}{\hat{\varrho}} p_{\sigma \hat{\varrho}} \nabla_{z_{i}}(\hat{\varrho} \hat{v} \cdot \hat{\nabla} \hat{\zeta}) \\
& \quad-p_{\sigma \hat{\varrho}} \hat{\varrho} \tilde{v}_{i t}-\frac{(\mu-\nu)}{\hat{\varrho}} p_{\sigma \hat{\varrho}} \nabla_{z_{i}} \hat{\varrho} \hat{\nabla} \cdot \tilde{v}, \quad i=1,2,3 .
\end{aligned}
$$

Multiplying the normal component of (3.34) by $\tilde{\varrho}_{\sigma n} J$, integrating over $\hat{\Omega}$ we obtain

$$
\begin{aligned}
& \frac{1}{2} \frac{d}{d t} \int_{\hat{\Omega}} \frac{p_{\sigma \hat{\varrho}}}{\hat{\varrho}} \tilde{\varrho}_{\sigma n}^{2} J d z+c_{0}\left\|\tilde{\varrho}_{\sigma n}\right\|_{0, \hat{\Omega}}^{2} \leq(\varepsilon+c d)\left\|\tilde{v}_{n n}\right\|_{0, \hat{\Omega}}^{2} \\
& \quad+\varepsilon\left\|\tilde{\varrho}_{\sigma n}\right\|_{0, \hat{\Omega}}^{2}+c\left(\left\|\tilde{v}_{z \tau}\right\|_{0, \hat{\Omega}}^{2}+\|\hat{v}\|_{1, \hat{\Omega}}^{2}+\left\|\tilde{v}_{t}\right\|_{0, \hat{\Omega}}^{2}+\left\|p_{\sigma}\right\|_{0, \hat{\Omega}}^{2}\right)+c X_{2}^{2}(\hat{\Omega})\left(1+X_{2}(\hat{\Omega})\right),
\end{aligned}
$$

where $d$ is from formula (3.21).

Now, we write $(3.22)_{1}$ in the form

$$
\hat{\varrho} \tilde{v}_{i t}-\mu \Delta \tilde{v}_{i}-\nu \nabla_{z_{i}} \nabla \cdot \tilde{v}=\hat{\nabla}_{i} \tilde{p}_{\sigma}+k_{3 i}-k_{6 i},
$$

where $k_{6 i}=\left(\mu \Delta \tilde{v}_{i}+\nu \nabla_{z_{i}} \nabla \cdot \tilde{v}\right)-\left(\mu \hat{\nabla}^{2} \tilde{v}_{i}+\nu \hat{\nabla}_{i} \hat{\nabla} \cdot \tilde{v}\right)$. 
Multiplying the third component of (3.36) by $\tilde{u}_{3 n n} J$ and integrating over $\hat{\Omega}$ yields

$$
\begin{aligned}
\frac{1}{2} \frac{d}{d t} \int_{\hat{\Omega}} \hat{\varrho} \tilde{v}_{3 n} J d z+c_{0}\left\|\tilde{v}_{3 n n}\right\|_{0, \hat{\Omega}}^{2} \leq & (\varepsilon+c d)\left\|\tilde{v}_{n n}\right\|_{0, \hat{\Omega}}^{2}+c\left(\left\|\tilde{v}_{z \tau}\right\|_{0, \hat{\Omega}}^{2}+\|\hat{v}\|_{1, \hat{\Omega}}^{2}\right. \\
& \left.+\left\|\tilde{v}_{t}\right\|_{1, \hat{\Omega}}^{2}+\left\|\tilde{\varrho}_{\sigma n}\right\|_{0, \hat{\Omega}}^{2}+\left\|p_{\sigma}\right\|_{0, \hat{\Omega}}^{2}\right) \\
& +c X_{2}^{2}(\hat{\Omega})\left(1+X_{2}(\hat{\Omega})\right) .
\end{aligned}
$$

For an interior subdomain the following estimate is obtained in the same way as (3.33)

$$
\begin{aligned}
& \frac{1}{2} \frac{d}{d t} \int_{\hat{\Omega}}\left(\bar{\varrho} \tilde{v}_{\xi}^{2}+\frac{p_{\sigma \bar{\varrho}}}{\bar{\varrho}} \tilde{\varrho}_{\sigma \xi}^{2}\right) A d \xi+c_{0}\|\tilde{v}\|_{2, \tilde{\Omega}}^{2} \leq \varepsilon\left(\left\|\hat{\varrho}_{\sigma \xi}\right\|_{0, \bar{\Omega}}^{2}+\left\|\tilde{v}_{\xi \xi}\right\|_{0, \bar{\Omega}}^{2}\right) \\
& \quad+c\left(\|\bar{v}\|_{1, \bar{\Omega}}^{2}+\left\|p_{\sigma}\right\|_{0, \Omega_{t}}^{2}\right)+c X_{2}^{2}(\tilde{\Omega})\left(1+X_{2}(\tilde{\Omega})\right),
\end{aligned}
$$

where

$$
X_{2}(\tilde{\Omega})=\sum_{i+k \leq 2}\left(\left\|\partial_{t}^{i} \bar{v}\right\|_{k, \tilde{\Omega}}+\left\|\partial_{t}^{i} \bar{\varrho}_{\sigma}\right\|_{k, \tilde{\Omega}}^{2}\right)+\int_{0}^{t}\|\bar{v}\|_{3, \tilde{\Omega}}^{2} d \tau+\|\bar{H}\|_{2, \tilde{\Omega}}^{2}
$$

and $A$ is the Jacobian of the transformation $x=x(\xi)$.

Finally, we have

$$
\frac{1}{2} \frac{d}{d t} \int_{\Omega} \varrho \bar{v}_{\xi}^{2} A d \xi \leq c\left(\|\bar{v}\|_{1, \Omega}^{2}+\left\|\bar{v}_{t}\right\|_{1, \Omega}^{2}\right)
$$

where we have used $(3.19)_{1}$.

Going back to the old variables $\xi$ in estimates (3.33), (3.35), (3.37) and summing them and (3.38) over all neighbourhoods of the partition of unity, using (3.25) and (3.40), assuming that $\varepsilon$ and $d$ are sufficiently small and passing to the variables $x$ we obtain (3.23).

This completes the proof.

LEMMA 3.6. Let $v, \varrho_{\sigma}$ be a sufficiently smooth solution of (3.2). Then

$$
\begin{aligned}
& \frac{1}{2} \frac{d}{d t} \int_{\Omega_{t}}\left(\varrho v_{x t}^{2}+\frac{p_{\sigma \varrho}}{\varrho} \varrho_{x t}^{2}\right) d x+c_{0}\left(\left\|v_{t}\right\|_{2, \Omega_{t}}^{2}+\left\|\varrho_{\sigma t}\right\|_{1, \Omega_{t}}^{2}\right) \\
& \quad \leq c\left(\|v\|_{1, \Omega_{t}}^{2}+\left\|v_{t}\right\|_{1, \Omega_{t}}^{2}+\left\|v_{t t}\right\|_{1, \Omega_{t}}^{2}+\left\|\varrho_{\sigma t}\right\|_{0, \Omega_{t}}^{2}+\left\|p_{\sigma}\right\|_{0, \Omega_{t}}^{2}\right)+c X_{2} Y_{2}\left(1+X_{2}^{2}\right),
\end{aligned}
$$

where $X_{2}$ is given by (3.10) and $Y_{2}$ is given by (3.18).

Proof. Differentiating problem (3.24) with respect to $t$ we get the following elliptic problem

$$
\begin{aligned}
& \mu \nabla_{v}^{2} \bar{v}_{t}+\nu \nabla_{v} \nabla_{v} \cdot \bar{v}_{t}-p_{\sigma \varrho} \nabla_{v} \bar{\varrho}_{\sigma t}=\bar{\varrho}_{t}+\varrho \bar{v}_{t t}-\nu\left(\nabla_{v} \nabla_{v}\right)_{t} \cdot \bar{v} \\
& +\mu_{1}\left(\stackrel{\frac{1}{H}}{H} \cdot \nabla_{v} \stackrel{\frac{1}{H}}{H}-\nabla_{v} \frac{\stackrel{1}{H}^{2}}{2}\right)-\mu\left(\nabla_{v}^{2}\right)_{t} \bar{v}+p_{\sigma \varrho \varrho} \varrho_{\sigma t} \nabla_{v} \varrho_{\sigma}+p_{\sigma \varrho}\left(\nabla_{v}\right)_{t} \varrho_{\sigma} \equiv K_{1} \quad \text { in } \Omega, \\
& \operatorname{div}_{v} \bar{v}_{t}=\operatorname{div}_{v} \bar{v}_{t} \\
& \text { in } \Omega \text {, } \\
& \mathbb{T}_{v}\left(\bar{v}_{t}, p_{\sigma t}\right) \bar{n}_{v}=-\left(\mathbb{T}_{v}\right)_{t}\left(\bar{v}, p_{\sigma}\right) \bar{n}_{v}-\mathbb{T}_{v}\left(\bar{v}, p_{\sigma}\right)\left(\bar{n}_{v}\right)_{t}
\end{aligned}
$$

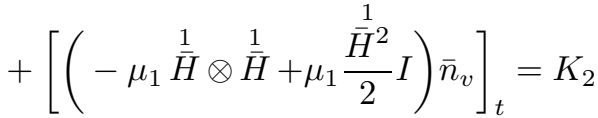

$$
\begin{aligned}
& \text { on } S \text {. }
\end{aligned}
$$


By the Agmon-Douglis-Nirenberg theory (see [1]) we have the estimate

$$
\left\|\bar{v}_{t}\right\|_{2, \Omega}^{2}+\left\|\bar{\varrho}_{\sigma t}\right\|_{1, \Omega}^{2} \leq c\left(\left\|K_{1}\right\|_{0, \Omega}^{2}+\left\|K_{2}\right\|_{1 / 2, S}^{2}+\left\|\operatorname{div}_{v} \bar{v}_{t}\right\|_{1, \Omega}^{2}\right)
$$

where

$$
\left\|K_{1}\right\|_{0, \Omega}^{2}+\left\|K_{2}\right\|_{1 / 2, S}^{2} \leq c\left(\left\|\bar{\varrho}_{\sigma \xi}\right\|_{0, \Omega}^{2}+\left\|\bar{v}_{t t}\right\|_{0, \Omega}^{2}+\left\|p_{\sigma}\right\|_{0, \Omega}^{2}\right)+X_{2}(\Omega) Y_{2}(\Omega)\left(1+X_{2}^{2}(\Omega)\right),
$$

$X_{2}(\Omega)$ is given by $(3.26)$ and

$$
Y_{2}(\Omega)=\sum_{\substack{i+k \leq 3 \\ i \leq 2}}\left\|\partial_{t}^{i} \bar{v}\right\|_{k, \Omega_{t}}^{2}+\left\|\bar{\varrho}_{\sigma}\right\|_{2, \Omega}^{2}+\left\|\bar{\varrho}_{\sigma t}\right\|_{2, \Omega}^{2}+\left\|\bar{\varrho}_{\sigma t t}\right\|_{1, \Omega}^{2} .
$$

By the arguments similar to considerations from the proof of Lemma 3.5 we conclude the proof.

LEMMA 3.7. Let $v, \varrho_{\sigma}$ be a sufficiently smooth solution of (3.2). Then

$$
\begin{aligned}
& \frac{1}{2} \frac{d}{d t} \int_{\Omega_{t}}\left(\varrho v_{x x}^{2}+\frac{p_{\sigma \varrho}}{\varrho} \varrho_{\sigma x x}^{2}\right) d x+c_{0}\left(\|v\|_{3, \Omega_{t}}^{2}+\left\|\varrho_{\sigma x}\right\|_{1, \Omega_{t}}^{2}\right) \\
& \leq c\left(\|v\|_{2, \Omega_{t}}^{2}+\left\|v_{t}\right\|_{1, \Omega_{t}}^{2}+\left\|\varrho_{\sigma x}\right\|_{0, \Omega_{t}}^{2}+\left\|p_{\sigma}\right\|_{0, \Omega_{t}}^{2}\right) \\
& \quad+\varepsilon\left\|v_{t}\right\|_{2, \Omega_{t}}^{2}+c X_{2} Y_{2}\left(1+X_{2}^{2}\right),
\end{aligned}
$$

where $X_{2}$ and $Y_{2}$ are given by (3.10) and (3.18), respectively and

$$
v_{x x}^{2}=\sum_{i, j, k=1}^{3} v_{i x_{j} x_{k}}^{2}, \quad \varrho_{\sigma x x}^{2}=\sum_{j, k=1}^{3} \varrho_{\sigma x_{j} x_{k}}^{2} .
$$

Proof. First, we consider problem (3.24). By the Agmon-Douglis-Nirenberg theory (see [1]) we have

$$
\|\bar{v}\|_{3, \Omega}^{2}+\left\|\bar{\varrho}_{\sigma}\right\|_{2, \Omega}^{2} \leq c\left(\left\|\bar{v}_{t}\right\|_{1, \Omega}^{2}+\|\operatorname{div} \bar{v}\|_{2, \Omega}^{2}\right)+c X_{2}(\Omega) Y_{2}(\Omega)\left(1+X_{2}^{2}(\Omega)\right),
$$

where $X_{2}(\Omega)$ and $Y_{2}(\Omega)$ are given by (3.26) and (3.41), respectively. Thus, to obtain (3.42) we have to estimate $\|\operatorname{div} \bar{v}\|_{2, \Omega}^{2}$ and $\frac{1}{2} \frac{d}{d t} \int_{\Omega_{t}}\left(\varrho v_{x x}^{2}+\frac{p_{\sigma \varrho}}{\varrho} \varrho_{\sigma x x}^{2}\right) d x$. To do this consider first boundary subdomains. Differentiate $(3.22)_{1}$ twice with respect to $\tau$, multiply the result by $\tilde{u}_{\tau \tau} J$ and integrate over $\hat{\Omega}$. Using the Korn inequality, continuity equation $(3.22)_{2}$, and boundary condition $(3.22)_{3}$ we get

$$
\begin{aligned}
\frac{1}{2} \frac{d}{d t} \int_{\hat{\Omega}}\left(\hat{\varrho} \tilde{v}_{\tau \tau}^{2}+\right. & \left.\frac{p_{\sigma \hat{\varrho}}}{\varrho} \tilde{\varrho}_{\sigma \tau \tau}^{2}\right) J d z+c_{0}\left\|\tilde{v}_{\tau \tau}\right\|_{1, \hat{\Omega}}^{2} \leq \varepsilon\left(\left\|\hat{\varrho}_{\sigma \tau \tau}\right\|_{0, \hat{\Omega}}^{2}+\left\|\tilde{v}_{\tau \tau}\right\|_{1, \hat{\Omega}}^{2}\right) \\
& +c\left(\|\hat{v}\|_{2, \hat{\Omega}}^{2}+\left\|\hat{\varrho}_{\sigma z}\right\|_{0, \hat{\Omega}}^{2}\right)+c X_{2}(\hat{\Omega}) Y_{2}(\hat{\Omega})\left(1+X_{2}^{2}(\hat{\Omega})\right),
\end{aligned}
$$

where $X_{2}(\hat{\Omega})$ is given by $(3.28)$ and

$$
Y_{2}(\hat{\Omega})=\sum_{\substack{i+k \leq 3 \\ i \leq 2}}\left\|\partial_{t}^{i} \bar{v}\right\|_{k, \hat{\Omega}}^{2}+\left\|\hat{\varrho}_{\sigma}\right\|_{2, \hat{\Omega}}^{2}+\left\|\hat{\varrho}_{\sigma t}\right\|_{2, \hat{\Omega}}^{2}+\left\|\hat{\varrho}_{\sigma t t}\right\|_{1, \hat{\Omega}}^{2}
$$

In the same way way we obtain the following estimate in an interior subdomain

$$
\begin{aligned}
\frac{1}{2} \frac{d}{d t} \int_{\hat{\Omega}}\left(\bar{\varrho} \tilde{v}_{\xi \xi}^{2}\right. & \left.+\frac{p_{\sigma \eta}}{\bar{\varrho}} \tilde{\varrho}_{\sigma \xi \xi}^{2}\right) A d \xi+c_{0}\|\tilde{v}\|_{3, \tilde{\Omega}}^{2} \leq \varepsilon\left(\left\|\tilde{\varrho}_{\sigma \xi \xi}\right\|_{0, \tilde{\Omega}}^{2}+\left\|\hat{v}_{\xi \xi \xi}\right\|_{0, \tilde{\Omega}}^{2}\right) \\
& +c\left(\|\bar{v}\|_{2, \tilde{\Omega}}^{2}+\left\|\bar{\varrho}_{\sigma \xi}\right\|_{0, \tilde{\Omega}}^{2}+\left\|p_{\sigma}\right\|_{0, \tilde{\Omega}}^{2}\right)+c X_{2}(\tilde{\Omega}) Y_{2}(\tilde{\Omega})\left(1+X_{2}^{2}(\tilde{\Omega})\right),
\end{aligned}
$$


where $X_{2}(\tilde{\Omega})$ is given by $(3.39)$ and

$$
Y_{2}(\tilde{\Omega})=\sum_{\substack{i+k \leq 3 \\ i \leq 2}}\left\|\partial_{t}^{i} \bar{v}\right\|_{k, \tilde{\Omega}}^{2}+\left\|\bar{\varrho}_{\sigma}\right\|_{2, \tilde{\Omega}}^{2}+\left\|\bar{\varrho}_{\sigma t}\right\|_{2, \tilde{\Omega}}^{2}+\left\|\bar{\varrho}_{\sigma t t}\right\|_{1, \tilde{\Omega}}^{2} .
$$

Now, we differentiate the third component of (3.34) by $\tau$, multiply the result by $\tilde{\varrho}_{\sigma n \tau} J$ and integrate over $\hat{\Omega}$. We get

$$
\begin{aligned}
& \frac{1}{2} \frac{d}{d t} \int_{\hat{\Omega}} \frac{p_{\sigma \hat{\varrho}}}{\hat{\varrho}} \tilde{\varrho}_{\sigma n \tau}^{2} J d z+\int_{\hat{\Omega}} p_{\sigma \hat{\varrho}}^{2} \tilde{\varrho}_{\sigma n \tau}^{2} J d z \leq \varepsilon\left\|\tilde{\varrho}_{\sigma n \tau}\right\|_{0, \hat{\Omega}}^{2}+c\left(\|\hat{v}\|_{2, \hat{\Omega}}^{2}+\left\|\hat{v}_{t}\right\|_{1, \hat{\Omega}}^{2}\right. \\
& \left.+\left\|\hat{\varrho}_{\sigma z}\right\|_{1, \hat{\Omega}}^{2}+\left\|p_{\sigma}\right\|_{0, \hat{\Omega}}^{2}\right)+c d\|\tilde{v}\|_{3, \hat{\Omega}}^{2}+c\left\|\tilde{v}_{z \tau \tau}\right\|_{0, \hat{\Omega}}^{2} \\
& +c X_{2}(\hat{\Omega}) Y_{2}(\hat{\Omega})\left(1+X_{2}^{2}(\hat{\Omega})\right),
\end{aligned}
$$

where $d$ is from formula (3.21).

In the same way we obtain

$$
\begin{aligned}
& \frac{1}{2} \frac{d}{d t} \int_{\hat{\Omega}} \frac{p_{\sigma \hat{\varrho}}}{\hat{\varrho}} \tilde{\varrho}_{\sigma n n}^{2} J d z+\int_{\hat{\Omega}} p_{\sigma \hat{\varrho}}^{2} \tilde{\varrho}_{\sigma n n}^{2} J d z \leq \varepsilon\left\|\tilde{\varrho}_{\sigma n n}\right\|_{0, \hat{\Omega}}^{2}+c\left(\|\hat{v}\|_{2, \hat{\Omega}}^{2}\right. \\
& \left.\quad+\left\|\hat{v}_{t}\right\|_{0, \hat{\Omega}}^{2}+\left\|\hat{\varrho}_{\sigma z}\right\|_{1, \hat{\Omega}}^{2}+\left\|p_{\sigma}\right\|_{0, \hat{\Omega}}^{2}\right)+c d\|\tilde{v}\|_{3, \hat{\Omega}}^{2}+c\left\|\tilde{v}_{z n \tau}\right\|_{0, \hat{\Omega}}^{2} \\
& \quad+c X_{2}(\hat{\Omega}) Y_{3}(\hat{\Omega})\left(1+X_{2}^{2}(\hat{\Omega})\right) .
\end{aligned}
$$

Next, differentiating the third component of (3.36) by $\tau$, multiplying by $\tilde{v}_{3 n n \tau} J$ and integrating over $\hat{\Omega}$ we have

$$
\begin{aligned}
& \frac{1}{2} \frac{d}{d t} \int_{\hat{\Omega}} \hat{\varrho} \tilde{v}_{3 n \tau}^{2} J d z+c_{0}\left\|\tilde{v}_{3 n n \tau}\right\|_{0, \hat{\Omega}}^{2} \leq \varepsilon\left\|\tilde{v}_{3 n n \tau}\right\|_{0, \hat{\Omega}}^{2}+c\left(\|\tilde{v}\|_{2, \hat{\Omega}}^{2}+\left\|\tilde{v}_{t}\right\|_{2, \tilde{\Omega}}^{2}\right. \\
& \left.\quad+\left\|\tilde{v}_{z \tau \tau}\right\|_{0, \hat{\Omega}}^{2}+\left\|\hat{\varrho}_{\sigma n \tau}\right\|_{0, \hat{\Omega}}^{2}+\left\|\hat{\varrho}_{\sigma z}\right\|_{0, \hat{\Omega}}^{2}+\left\|p_{\sigma}\right\|_{0, \hat{\Omega}}^{2}\right)+c d\|\hat{v}\|_{3, \hat{\Omega}}^{2}+\varepsilon\left\|\tilde{v}_{t}\right\|_{2, \hat{\Omega}}^{2} \\
& \quad+c X_{2}(\hat{\Omega}) Y_{2}(\hat{\Omega})\left(1+X_{2}^{2}(\hat{\Omega})\right) .
\end{aligned}
$$

In order to estimate $\left\|(\operatorname{div} \tilde{v})_{n n}\right\|_{0, \hat{\Omega}}^{2}$ rewrite equation $(3.22)_{1}$ in the form

$$
\begin{aligned}
(\nu+\mu) \nabla_{z_{i}} \operatorname{div} \tilde{v}= & -\mu\left(\Delta \tilde{v}_{i}-\nabla_{z_{i}} \operatorname{div} \tilde{v}\right)+\hat{\varrho} \tilde{v}_{i t}-k_{3 i} \\
& +\left(\mu \Delta \tilde{v}_{i}+\nu \nabla_{z_{i}} \operatorname{div} \tilde{v}-\mu \hat{\nabla}^{2} \tilde{v}_{i}-\nu \hat{\nabla}_{i} \hat{\nabla} \cdot \tilde{v}\right)+\mu_{1}\left(-\tilde{H} \hat{\nabla} \hat{H}_{i}+\left(\hat{\nabla} \hat{\nabla}_{i} \hat{H}\right) \stackrel{1}{\tilde{H}}\right) \\
& +p_{1} \hat{\varrho}_{\sigma} \hat{\nabla}_{i} \hat{\zeta}+\hat{\zeta} p_{\sigma} \hat{\vartheta}_{i} \hat{\varrho}_{\sigma}, \quad i=1,2,3 .
\end{aligned}
$$

Differentiating the third component of (3.49) with respect to $n$ gives

$$
\begin{aligned}
\left\|(\operatorname{div} \tilde{v})_{n n}\right\|_{0, \hat{\Omega}}^{2} \leq & c d\left\|\tilde{v}_{n n n}\right\|_{0, \hat{\Omega}}^{2}+c\left(\left\|\tilde{v}_{\tau}\right\|_{2, \hat{\Omega}}^{2}+\|\hat{v}\|_{2, \hat{\Omega}}^{2}+\left\|\tilde{v}_{t}\right\|_{1, \hat{\Omega}}^{2}\right. \\
& \left.+\left\|\hat{\varrho}_{\sigma z}\right\|_{1, \hat{\Omega}}^{2}+\left\|p_{\sigma}\right\|_{0, \hat{\Omega}}^{2}\right)+c X_{2}(\hat{\Omega}) Y_{2}(\hat{\Omega}) .
\end{aligned}
$$

To obtain an estimate for $\left\|\tilde{v}_{\tau}\right\|_{2, \hat{\Omega}}^{2}$ consider the following elliptic problem

$$
\begin{aligned}
& \mu \hat{\nabla}^{2} \tilde{v}+\nu \hat{\nabla} \hat{\nabla} \cdot \tilde{v}-p_{\sigma \hat{\varrho}} \hat{\varrho}_{\sigma}=\hat{\varrho} \tilde{v}_{t}+\left(p_{1}-p_{\sigma} \hat{\varrho}\right) \hat{\varrho}_{\sigma} \hat{\nabla} \hat{\zeta} \\
& \quad+\mu_{1}\left(-\stackrel{1}{\tilde{H}} \cdot \stackrel{1}{\hat{H}}+\frac{1}{2}\left(\hat{\nabla}(\stackrel{1}{\tilde{H}} \hat{H})-(\hat{\nabla} \hat{\zeta}) \hat{H}^{2}\right)\right)+\hat{\nabla} \cdot \hat{\mathbb{B}}(\hat{v}, \hat{\zeta})+\hat{\mathbb{T}}\left(\hat{v}, p_{\sigma}\right) \cdot \hat{\nabla} \hat{\zeta}, \\
& \hat{\nabla} \cdot \tilde{v}=\hat{\nabla} \cdot \tilde{v} \\
& \hat{\mathbb{T}}\left(\tilde{v}, \tilde{p}_{\sigma}\right) \hat{n}=k_{5},
\end{aligned}
$$


where $\hat{\nabla} \cdot \hat{\mathbb{B}}(\hat{v}, \hat{\zeta})=\left\{\hat{\nabla}_{j} \hat{\mathbb{B}}_{i j}(\hat{v}, \hat{\zeta})\right\}_{i=1,2,3}, \hat{\mathbb{T}}\left(\hat{v}, p_{\sigma}\right) \cdot \hat{\nabla} \hat{\zeta}=\left\{\hat{T}_{v i j}\left(\hat{v}, p_{\sigma}\right) \hat{\nabla}{ }_{j} \hat{\zeta}\right\}_{i=1,2,3}$.

Differentiating (3.51) with respect to $\tau$ and next using the Agmon-Douglis-Nirenberg theory we get

$$
\begin{aligned}
\left\|\tilde{v}_{\tau}\right\|_{2, \hat{\Omega}}^{2}+\left\|\hat{\varrho}_{\sigma \tau}\right\|_{1, \hat{\Omega}}^{2} \leq & c\left(\left\|\tilde{v}_{\tau \tau}\right\|_{1, \hat{\Omega}}^{2}+\left\|\hat{v}_{3 n n \tau}\right\|_{0, \hat{\Omega}}^{2}+\|\hat{v}\|_{2, \hat{\Omega}}^{2}+\left\|\hat{v}_{t}\right\|_{1, \hat{\Omega}}^{2}\right. \\
& \left.+\left\|\hat{\varrho}_{\sigma z}\right\|_{0, \hat{\Omega}}^{2}+\left\|p_{\sigma}\right\|_{0, \hat{\Omega}}^{2}\right)+c X_{2}(\hat{\Omega}) Y_{2}(\hat{\Omega})\left(1+X_{2}(\hat{\Omega})\right) .
\end{aligned}
$$

Finally, we have

$$
\frac{1}{2} \frac{d}{d t} \int_{\Omega} \varrho \bar{v}_{\xi \xi}^{2} A d \xi \leq c\|\bar{v}\|_{2, \Omega}^{2}+c\left\|\bar{v}_{t}\right\|_{1, \Omega}^{2}
$$

Going back to the old variables $\xi$ in estimates $(3.44),(3.46),(3.49),(3.50),(3.52)$ and summing them and (3.45) over all neighbourhoods of the partition of unity, using (3.43) and (3.53), assuming that $\varepsilon$ and $d$ are sufficiently small and passing to the variables $x$ we obtain (3.44). This concludes the proof.

In [7] we proved the following lemmas for problem (1.1)-(1.7):

LEMMA 3.8. For a sufficiently smooth solution $\left(v, \varrho_{\sigma}, H\right)$ of $(1.1)-(1.7)$, we have

$$
\frac{d}{d t}\|H\|_{0, \Pi_{t}}^{2}+\|H\|_{1, \Pi_{t}}^{2} \leq c\|H\|_{1, \Pi_{t}}^{2}\|v\|_{1, \Pi_{t}}^{2} .
$$

LEMMA 3.9. For a sufficiently smooth solution $\left(v, \varrho_{\sigma}, H\right)$ of $(1.1)-(1.7)$, we have

$$
\frac{d}{d t}\left\|H_{t}\right\|_{0, \Pi_{t}}^{2}+\left\|H_{t}\right\|_{1, \Pi_{t}}^{2} \leq c\left(\left\|H_{t}\right\|_{1, \Pi_{t}}^{2}\|v\|_{1, \Pi_{t}}^{2}+\left\|v_{t}\right\|_{1, \Pi_{t}}^{2}\|H\|_{1, \Pi_{t}}^{2}\right) .
$$

LEMMA 3.10. For a sufficiently smooth solution $\left(v, \varrho_{\sigma}, H\right)$ of (1.1)-(1.7), we have

$$
\begin{aligned}
\frac{d}{d t}\left\|H_{t t}\right\|_{0, \Pi_{t}}^{2}+\left\|H_{t t}\right\|_{1, \Pi_{t}}^{2} \leq & c\left(\left\|H_{t t}\right\|_{1, \Pi_{t}}^{2}\|v\|_{1, \Pi_{t}}^{2}+\left\|v_{t t}\right\|_{1, \Pi_{t}}^{2}\|H\|_{1, \Pi_{t}}^{2}\right. \\
& \left.+\left\|v_{t}\right\|_{1, \Pi_{t}}^{2}\left\|H_{t}\right\|_{1, \Pi_{t}}^{2}\right) .
\end{aligned}
$$

LEMma 3.11. For a sufficiently smooth solution $\left(v, \varrho_{\sigma}, H\right)$ of (1.1)-(1.7), we have

$$
\begin{aligned}
& \frac{d}{d t}\left\|H_{t}\right\|_{1, \Pi_{t}}^{2}+\left\|H_{t}\right\|_{2, \Pi_{t}}^{2} \leq c \varphi(a)\left[\left\|H_{t t}\right\|_{1, \Pi_{t}}^{2}+\left\|H_{t}\right\|_{2, \Pi_{t}}^{2}\|v\|_{1, \Pi_{t}}^{2}+\|H\|_{2, \Pi_{t}}^{2}\left(\|v\|_{3, \Pi_{t}}^{2}\right.\right. \\
& \left.\quad+\left\|v_{t}\right\|_{2, \Pi_{t}}^{2}+\|v\|_{2, \Pi_{t}}^{4}\right)+\|v\|_{3, \Pi_{t}}^{2}\left(\left\|H_{t}\right\|_{1, \Pi_{t}}^{2}+\|H\|_{2, \Pi_{t}}^{2}\|v\|_{2, \Pi_{t}}^{2}+\|v\|_{2, \Pi_{t}}^{2}+\left\|v_{t}\right\|_{2, \Pi_{t}}^{2}\right) \\
& \left.\quad+\|v\|_{2, \Pi_{t}}^{2}+a^{2}\|H\|_{3, \Pi_{t}}^{2}\right] .
\end{aligned}
$$

LEMma 3.12. For a sufficiently smooth solution $\left(v, \varrho_{\sigma}, H\right)$ of (1.1)-(1.7), we have

$$
\begin{aligned}
\frac{d}{d t}\|H\|_{2, \Pi_{t}}^{2}+\|H\|_{3, \Pi_{t}}^{2} \leq & c \varphi(a)\left[\|H\|_{2, \Pi_{t}}^{2}\|v\|_{2, \Pi_{t}}^{2}+\|v\|_{2, \Pi_{t}}^{2}+\|H\|_{2, \Pi_{t}}^{2}\|v\|_{3, \Pi_{t}}^{2}+\right. \\
& \left.+\left\|H_{t}\right\|_{2, \Pi_{t}}^{2}+\|H\|_{3, \Pi_{t}}^{2}\|v\|_{2, \Pi_{t}}^{2}\right] .
\end{aligned}
$$

LEMMA 3.13. For sufficiently smooth solution $\left(v, \varrho_{\sigma}, H\right)$ of (1.1)-(1.7), we have

$$
\frac{d}{d t}\|H\|_{1, \Pi_{t}}^{2} \leq c\left(\|H\|_{1, \Pi_{t}}^{2}+\varepsilon\left\|H_{t}\right\|_{1, \Pi_{t}}^{2}+\|H\|_{2, \Pi_{t}}^{2}\|v\|_{0, \Pi_{t}}^{2}\right) .
$$

Now let $\stackrel{2}{H}=H_{*}$ on $B$, then from Lemmas 3.1-3.13 and inequalities

$$
\begin{aligned}
\left\|\varrho_{\sigma t t}\right\|_{1, \Omega_{t}}^{2} & \leq c\left\|v_{t}\right\|_{2, \Omega_{t}}^{2}+c\left(\left\|\varrho_{\sigma t}\right\|_{2, \Omega_{t}}^{2}\|v\|_{2, \Omega_{t}}^{2}+\left\|\varrho_{\sigma}\right\|_{2, \Omega_{t}}^{2}\left\|v_{t}\right\|_{2, \Omega_{t}}^{2}\right), \\
\left\|\varrho_{\sigma t}\right\|_{2, \Omega_{t}}^{2} & \leq c\|v\|_{3, \Omega_{t}}^{2}+c X_{2} Y_{2}\left(1+X_{2}\right),
\end{aligned}
$$


(which follow from equations $(3.2)_{2}$ and $(3.19)_{2}$, respectively) we get

THEOREM 3.1. Let $\nu>\frac{1}{3} \mu>0$ and let Remarks 3.1 and 3.2 be satisfied. Then for a sufficiently smooth solution $v, \varrho_{\sigma}, H$ of problem (1.1)-(1.7) we have

$$
\frac{d \bar{\phi}}{d t}+c_{0} \Phi \leq c_{1}\left(\phi+\int_{0}^{t}\|v\|_{3, \Omega_{\tau}}^{2} d \tau\right)\left[1+\left(\phi+\int_{0}^{t}\|v\|_{3, \Omega_{\tau}}^{2} d \tau\right)^{2}\right] \Phi+c_{2} \Psi \quad \text { for } t \leq T
$$

where

$$
\begin{aligned}
\bar{\phi}(t)= & \int_{\Omega_{t}} \varrho \sum_{0 \leq|\alpha|+i \leq 2}\left|D_{x}^{\alpha} \partial_{t}^{i} v\right|^{2} d x+\int_{\Omega_{t}} \frac{p_{1}}{\varrho} \varrho_{\sigma}^{2} d x \\
& +\int_{\Omega_{t}} \frac{p_{\sigma \varrho}}{\varrho} \sum_{1 \leq|\alpha|+i \leq 2}\left|D_{x}^{\alpha} \partial_{t}^{i} p_{\sigma}\right|^{2} d x+\sum_{i+k \leq 2}\left\|\partial_{t}^{i} H\right\|_{k, \Pi_{t}}^{2}, \\
\phi(t)= & \sum_{i+k \leq 2}\left(\left\|\partial_{t}^{i} v\right\|_{k, \Omega_{t}}^{2}+\left\|\partial_{t}^{i} H\right\|_{k, \Pi_{t}}^{2}+\left\|\partial_{t}^{i} \varrho_{\sigma}\right\|_{k, \Omega_{t}}^{2}\right), \\
\Phi(t)= & \left\|\varrho_{\sigma}\right\|_{2, \Omega_{t}}^{2}+\left\|\varrho_{\sigma t}\right\|_{2, \Omega_{t}}^{2}+\left\|\varrho_{\sigma t t}\right\|_{1, \Omega_{t}}^{2}+\sum_{\substack{i+k \leq 3 \\
i \leq 2}}\left(\left\|\partial_{t}^{i} v\right\|_{k, \Omega_{t}}^{2}+\left\|\partial_{t}^{i} H\right\|_{k, \Pi_{t}}^{2}\right) \\
\Psi(t)= & \left\|p_{\sigma}\right\|_{0, \Omega_{t}}^{2}+\left\|H_{*}\right\|_{3, B}^{2}+\left\|H_{*}\right\|_{1, B}^{4}+\left\|H_{* t}\right\|_{2, B}^{2}\left(1+\left\|H_{* t}\right\|_{2, B}^{2}\right)+\left\|H_{* t t}\right\|_{1, B}^{2},
\end{aligned}
$$

$c_{i}(i=1,2)$ are positive constants depending on $\varphi_{*}, \varphi^{*}, \mu, \nu, \int_{0}^{t}\|v\|_{3, \Omega_{\tau}}^{2} d \tau,\|S\|_{\frac{5}{2}}, T$ and constants of the imbedding theorems and the Korn inequalities; $c_{0}<1$ is a positive constant depending on $\mu$ and $\nu ; \varrho_{\sigma}$ and $p_{\sigma}$ are given by (3.1).

4. Global existence. Now, let $\bar{\phi}(t), \phi(t)$ and $\Phi(t)$ be defined by (3.61). Introduce the spaces

$$
\mathcal{N}(t)=\left\{\left(v, \varrho_{\sigma}, H\right): \phi(t)<\infty\right\}
$$

and

$$
\mathcal{M}(t)=\left\{\left(v, \varrho_{\sigma}, H\right): \phi(t)+\int_{0}^{t} \Phi(\tau) d \tau<\infty\right\} .
$$

Notice that $\left(v, \varrho_{\sigma}, H\right) \in \mathcal{N}(t)$ iff $\bar{\phi}(t)<\infty$ and $\left(v, \varrho_{\sigma}, H\right) \in \mathcal{M}(t)$ iff $\bar{\phi}(t)+\int_{0}^{t} \phi(\tau) d \tau<\infty$. Moreover,

$$
c^{\prime} \phi(t) \leq \bar{\phi}(t) \leq c^{\prime \prime} \phi(t)
$$

where $c^{\prime}, c^{\prime \prime}>0$ are constants depending on $\varphi_{*}, \varphi^{*}$ given by $(2.5)$.

From Theorem 2.1 and (4.1) we get

Lemma 4.1. Let $\left(v(0), \varrho_{\sigma}(0), H(0)\right) \in \mathcal{N}(0)$ and $\varphi(0)<\varepsilon_{1}$. Then $\left(v(t), \varrho_{\sigma}(t), H(t)\right) \in$ $\mathcal{M}(t), t<T$ where $T$ is the time of local existence and

$$
\begin{aligned}
\bar{\phi}(t)+\int_{0}^{t} \Phi(\tau) d \tau \leq & c\left(\varepsilon_{1}+\int_{0}^{t}\left(\left\|E_{*}\right\|_{0, B}^{2}+\left\|E_{* t}\right\|_{0, B}^{2}+\left\|H_{*}\right\|_{3, B}^{2}+\left\|H_{* t}\right\|_{2, B}^{2}+\right.\right. \\
& \left.\left.+\left\|H_{* t t}\right\|_{0, B}^{2}\right) d \tau\right) \equiv c\left(\varepsilon_{1}+\beta\right) .
\end{aligned}
$$

Proof. From inequality

$$
\|\bar{H}\|_{2, \Pi^{t}}^{2} \leq c\left(\varepsilon\left\|\bar{H}_{t}\right\|_{2,2,2, \Pi^{t}}^{2}+c(\varepsilon)\|\bar{H}\|_{2,2,2, \Pi^{t}}^{2}+\|\bar{H}(0)\|_{2, \Pi}^{2}\right)
$$

and Theorem 2.1 we get (4.2). Next we prove 
Lemma 4.2. Assume that there exists a local solution of (1.1)-(1.7) in $\mathcal{M}(t), 0 \leq t \leq T$ with initial data in $\mathcal{N}(0)$ sufficiently small and

$$
\alpha(t)=\left\|p_{\sigma}\right\|_{0, \Omega_{t}}^{2}+\left\|H_{*}\right\|_{3, B}^{2}+\left\|H_{*}\right\|_{1, B}^{4}+\left\|H_{* t}\right\|_{2, B}^{2}\left(1+\left\|H_{* t}\right\|_{2, B}^{2}\right)+\left\|H_{* t t}\right\|_{1, B}^{2} \leq e^{-\mu t}
$$

$0 \leq t \leq T$, where $\mu>\frac{1}{2}$. Then there exist constants $\mu_{1}>1, \mu_{2}>0$ such that

$$
\bar{\phi}(t) \leq \mu_{1} e^{-\mu_{2} t}\left(\bar{\phi}(0)+\frac{c_{2}}{\mu-\mu_{2}}\right)
$$

if $\mu>\mu_{2}$.

Proof. Consider inequality (3.63) and assume that $\varepsilon_{1}+\beta$ from (4.2) (see Lemma 4.1) is so small that

$$
c_{1}\left(\phi+\int_{0}^{t}\|v\|_{3, \Omega_{\tau}}^{2} d \tau\right)\left[1+\left(\phi+\int_{0}^{t}\|v\|_{3, \Omega_{\tau}}^{2} d \tau\right)^{2}\right]<\frac{c_{0}}{4} .
$$

Then inequality (3.61) implies

$$
\begin{aligned}
& \frac{d \bar{\phi}}{d t}+\frac{3}{4} c_{0} \Phi \\
& <c_{2}\left(\left\|p_{\sigma}\right\|_{0, \Omega_{t}}^{2}+\left\|H_{*}\right\|_{3, B}^{2}+\left\|H_{*}\right\|_{1, B}^{4}+\left\|H_{* t}\right\|_{2, B}^{2}\left(1+\left\|H_{* t}\right\|_{2, B}^{2}\right)+\left\|H_{* t t}\right\|_{1, B}^{2}\right) .
\end{aligned}
$$

Applying the same argument as in the proof of Lemma 6.2 of [19] yields

$$
\left\|p_{\sigma}\right\|_{0, \Omega_{t}}^{2} \leq \varepsilon\left(\left\|p_{\sigma x}\right\|_{0, \Omega_{t}}^{2}+\left\|v_{x x}\right\|_{0, \Omega_{t}}^{2}\right)+c(\varepsilon)\left(\|v\|_{0, \Omega_{t}}^{2}+\left\|v_{t}\right\|_{0, \Omega_{t}}^{2}\right) .
$$

Since

$$
\left\|p_{\sigma x}\right\|_{0, \Omega_{t}}^{2} \leq c_{4}\left\|\varrho_{\sigma x}\right\|_{0, \Omega_{t}}^{2}
$$

inequalities (4.5) and (4.6) imply for sufficiently small $\varepsilon$

$$
\begin{aligned}
\frac{d \bar{\phi}}{d t}+\frac{3}{4} c_{0} \Phi<c_{5}\left(\|v\|_{0, \Omega_{t}}^{2}+\left\|v_{t}\right\|_{0, \Omega_{t}}^{2}\right) & +c_{2}\left(\left\|H_{*}\right\|_{3, B}^{2}+\left\|H_{*}\right\|_{1, B}^{4}\right. \\
& \left.+\left\|H_{* t}\right\|_{2, B}^{2}\left(1+\left\|H_{* t}\right\|_{2, B}^{2}\right)+\left\|H_{* t t}\right\|_{1, B}^{2}\right) .
\end{aligned}
$$

Now, multiplying (3.16) by a constant $c_{6}$ so large that $c_{0} c_{6}-c_{5}>0$ and $c_{6}>1$, adding to (4.7) and using Lemma 4.1 we obtain

$$
\begin{aligned}
\frac{d}{d t}\left(\bar{\phi}+c_{6} J\right) & +\frac{3}{4} c_{0} \Phi+\left(c_{0} c_{6}-c_{5}\right)\left(\|v\|_{1, \Omega_{t}}^{2}+\left\|v_{t}\right\|_{1, \Omega_{t}}^{2}+\left\|\varrho_{\sigma t}\right\|_{0, \Omega_{t}}^{2}\right)<c_{7}\left(\beta+c \varepsilon_{1}\right) \phi \\
& +c_{2}\left(\left\|H_{*}\right\|_{3, B}^{2}+\left\|H_{*}\right\|_{3, B}^{4}+\left\|H_{* t}\right\|_{2, B}^{2}\left(1+\left\|H_{* t}\right\|_{2, B}^{2}\right)+\left\|H_{* t t}\right\|_{1, B}^{2}\right)
\end{aligned}
$$

where $J=\frac{1}{2} \int_{\Omega_{t}}\left[\varrho\left(v^{2}+v_{t}^{2}\right)+\frac{p_{1}}{\varrho} \varrho_{\sigma}^{2}+\frac{p_{\sigma \varrho}}{\varrho} \varrho_{\sigma t}^{2}\right] d x$. Since $\frac{\bar{\phi}}{c^{\prime \prime}} \leq \phi \leq \Phi$ and $\bar{\phi} \geq J$ for sufficiently small $\beta+c \varepsilon_{1}\left(\beta+c \varepsilon_{1}\right.$ so small that $\left.c_{7}\left(\beta+c \varepsilon_{1}\right)<\frac{1}{4} c_{0}\right)$ inequality (4.8) implies

$$
\frac{d}{d t}\left(\bar{\phi}+c_{6} J\right)+c_{8}\left(\bar{\phi}+c_{6} J\right) \leq c_{2} e^{-\mu t},
$$

where $c_{8}=\frac{c_{0}}{4 c^{\prime \prime} c_{6}}\left(c^{\prime \prime}>0\right.$ is a constant from (4.1)).

Inequality (4.9) yields (4.3) with $\mu_{1}=c_{6}+1$ and $\mu_{2}=c_{8}$. This completes the proof of Lemma 4.2 .

Lemma 4.3. Let the assumptions of Lemma 4.2 be satisfied and $\bar{\phi}(0)<\varepsilon_{1}$. Then $\bar{\phi}(T) \leq$ $\varepsilon_{1}$, where $T>0$ is the time of local existence. 
Proof. If $\mu>0$ and $T$ are sufficiently large then from Lemma 4.2

$$
\bar{\varphi}(T) \leq \mu_{1} e^{-\mu_{2} T}\left(\bar{\varphi}(0)+\frac{c_{2}}{\mu-\mu_{2}}\right) \leq \bar{\varphi}(0) .
$$

Proof of Main Theorem. The theorem is proved step by step using local existence in a fixed time interval. Under the assumptions that

$$
\left(v(0), \varrho_{\sigma}(0), H(0)\right) \in \mathcal{N}(0) .
$$

Theorem 2.1 and Lemma 4.1 yield local existence of solutions of (1.1)-(1.7).

By (4.8), Lemma 4.1 implies that the local solution belongs to $\mathcal{M}(t), t \leq T$. For small $\varepsilon_{1}$ and $\beta$ the existence time $T$ is correspondingly large, so we can assume it is a fixed positive number. The constants in those theorems depend on $\Omega_{t}$ and shape of $S_{t}$ and $\int_{0}^{t}\|v\|_{3, \Omega_{\tau}}^{2} d \tau$, so generally they are functions of $t$.

But in view of (4.1) with sufficiently small $\varepsilon_{1}, \beta$ we obtain

$$
\left|\int_{0}^{t} v d \tau\right| \leq c \beta \quad t \in[0, T] .
$$

Hence from the relation

$$
x=\xi+\int_{0}^{t} v(x(\xi, \tau), \tau) d \tau, \quad \xi \in S, \quad t \leq T,
$$

for sufficiently small $\varepsilon_{1}, \beta$ and fixed $T$, the shape of $\Omega_{t}, t \leq T$ does not change too much, so the constants from the immbeding theorems can be chosen independent of time. Now we wish to extend the solution to the interval $[T, 2 T]$. Using Lemma 4.3 we can prove the existence of local solution in $\mathcal{M}(t), T \leq t \leq 2 T$. To prove

$$
\bar{\phi}(2 T) \leq \varepsilon_{1},
$$

we need inequality (3.61) where the constans depend on the constants from the imbedding theorems and Korn inequalities for $t \in[T, 2 T]$. Therefore we have to show that the shape of $S_{t}$ and $\int_{0}^{t}\|v\|_{3, \Omega_{\tau}}^{2} d \tau, \quad t \leq 2 T$, do not change more than for $t \leq T$. Assume that there exists a local solution in the interval $[0, k T]$. Then in view of Lemma 4.2 , we have for $t \in[0, k T]$

$$
\begin{aligned}
& \left|\int_{0}^{t} v d x\right| \leq\left|\int_{0}^{t}\|v\|_{2, \Omega_{\tau}} d \tau\right| \\
& \leq c_{1} \sum_{i=0}^{k-1} \int_{i T}^{(i+1) T}\|v\|_{2, \Omega_{t}} d t \leq c_{1} T^{\frac{1}{2}} \sum_{i=0}^{k-1}\left(\int_{i T}^{(i+1) T}\|v\|_{2, \Omega_{t}}^{2} d t\right)^{\frac{1}{2}} \\
& \leq c_{1} T^{\frac{1}{2}} \sum_{i=0}^{k-1}\left(\int_{i T}^{(i+1) T} \bar{\phi}(t) d t\right)^{\frac{1}{2}} \\
& \leq c_{1}\left(\frac{T}{\mu_{2}}\right)^{\frac{1}{2}} \sum_{i=0}^{k-1}\left[\frac{c_{2}}{\mu-\mu_{2}} e^{-i T \mu}+\bar{\phi}(i T)\right]^{\frac{1}{2}} \\
& \leq c\left(\frac{T}{\mu_{2}}\right)^{\frac{1}{2}} \frac{1}{1-e^{-\mu_{2} T / 2}}\left[\frac{1}{\left(\mu-\mu_{2}\right)^{\frac{1}{2}}}\left(1+\frac{1}{1-e^{-\mu_{2} T / 2}}\right)+\left(\bar{\phi}(0) \mu_{1}\right)^{\frac{1}{2}}\right]
\end{aligned}
$$


and

$$
\begin{aligned}
\int_{0}^{t}\|v\|_{3, \Omega_{\tau}}^{2} d \tau & \leq \sum_{i=0}^{k-1} \int_{i T}^{(i+1) T}\|v\|_{3, \Omega_{\tau}}^{2} d \tau \leq \sum_{i=0}^{k-1} \int_{i T}^{(i+1) T} \Phi d t \\
& \leq \frac{1}{\mu_{2}} \sum_{i=0}^{k-1}\left(\frac{c_{2}}{\mu} e^{-\mu i T}+\mu_{2} \bar{\phi}(i T)\right) \\
& \leq \frac{c}{\mu_{2}\left(1-e^{-\mu_{2} T}\right)}\left(\frac{1}{\mu}+\mu_{1} \bar{\phi}(0)+\frac{1}{\left(\mu-\mu_{2}\right)\left(1-e^{-\mu_{2} T}\right)}\right)
\end{aligned}
$$

since from (4.9)

$$
A((i+1) T) \leq \frac{c_{2}}{\mu-\mu_{2}} e^{-\mu_{2}(i+1) T}+A(i T) e^{-\mu_{2} T},
$$

where $A=\bar{\phi}+c_{6} I$, then we have

$$
\begin{aligned}
\sum_{i=0}^{k-1} \bar{\phi}(i T) & \leq \sum_{i=0}^{k-1} A(i T) \leq \frac{\mu_{1} \bar{\phi}(0)}{1-e^{-\mu_{2} T}}+\frac{c_{2}}{\mu-\mu_{2}} \frac{e^{-\mu_{2} T}}{1-e^{-\mu_{2} T}}+\frac{c_{2}}{\mu-\mu_{2}} \frac{e^{-2 \mu_{2} T}}{1-e^{-\mu_{2} T}} \\
& +\cdots+\frac{c_{2}}{\mu-\mu_{2}} \frac{e^{-n \mu_{2} T}}{1-e^{-\mu_{2} T}}+\cdots \leq \frac{1}{1-e^{\mu_{2} T}}\left(\mu_{1} \bar{\phi}(0)+\frac{c_{2} e^{-\mu_{2} T}}{\left(\mu-\mu_{2}\right)\left(1-e^{-\mu_{2} T}\right)}\right)
\end{aligned}
$$

also

$$
\int_{i T}^{(i+1) T} A(t) d t \leq \frac{c_{2}}{\left(\mu-\mu_{2}\right) \mu_{2}} e^{-\mu i T}+\frac{1}{\mu_{2}} A(i T)
$$

and

$$
A((i+1) T)+\mu_{2} \int_{i T}^{(i+1) T} A(t) d t \leq \frac{c_{2}}{\mu} e^{-\mu i T}+A(i T), \quad i=0,1, \ldots, k-1 .
$$

Taking $k=2, \varepsilon_{1}$ sufficiently small and $\mu$ sufficiently large we see that $\int_{0}^{t} v(x(\xi, \tau), \tau) d \tau$ is small for any $t \in[0,2 T]$, so (4.14) implies that the shape of $S_{t}$ and $\int_{0}^{t}\|v\|_{3, \Omega_{\tau}}^{2} d \tau$ change no more than in $[0, T]$, and then the differential inequality (3.62) can also be shown for this interval with the same constants. Hence in view of Lemma 4.1 the solutions of $(1.1)-(1.7)$ belongs to $\mathcal{M}(t), t \in[T, 2 T]$. Next Lemmas 4.1-4.3 imply (4.14).

Repeating the above considerations for the intervals $[k T,(k+1) T], k \geq 2$, we prove the existence for all $t \in \mathbb{R}_{+}$.

\section{Korn inequality}

Lemma 5.1. Let $\Omega_{t} \subset \mathbb{R}^{3}$ be a bounded domain. Let $\left(v, \varrho_{\sigma}\right)$ be a solution of $(1.1)_{1},(1.1)_{2}$, $(1.5)_{1}$ and $f=\int_{\Omega} v_{0} d x=\int_{\Omega} \varrho_{0} v_{0} \cdot \varphi_{i} d x, i=1,2,3$ and

$$
\mathbb{E}_{\Omega_{t}}(v)=\int_{\Omega_{t}}\left(\partial_{x_{i}} v_{j}+\partial_{x_{j}} v_{i}\right)^{2} d x<\infty
$$

Then there exists a constant $c>0$ such that

$$
\|v\|_{1, \Omega_{t}}^{2} \leq c\left(\mathbb{E}_{\Omega_{t}}(v)+\left(\int_{\Omega_{t}}\left|\left(\varrho-\varrho_{e}\right)\right||v| d x\right)^{2}\right)
$$


Proof. Introduce a function $u$ by

$$
u=\sum_{i=1}^{3} b_{i} \varphi_{i}(x)+v
$$

where $\varphi_{i}=(x-\bar{x}) \times e_{i}, \quad \bar{x}=\frac{1}{\left|\Omega_{t}\right|}\left(\int_{\Omega_{t}} x_{1} d x, \int_{\Omega_{t}} x_{2} d x, \int_{\Omega_{t}} x_{3} d x\right), e_{i}=\left(\delta_{i 1}, \delta_{i 2}, \delta_{i 3}\right)$, $i=1,2,3$.

Define $b=\left(b_{1}, b_{2}, b_{3}\right)$ by

$$
b=\frac{1}{2\left|\Omega_{t}\right|} \int_{\Omega_{t}} \operatorname{rot} v d x .
$$

Since rot $\varphi_{i}=2 e_{i}, i=1,2,3$, equations (5.3) and (5.4) imply

$$
\int_{\Omega_{t}} \operatorname{rot} u d x=0
$$

From (5.4) we have $\int_{\Omega_{t}} \varphi_{i} d x=0, i=1,2,3$ so

$$
\int_{\Omega_{t}} u d x=\int_{\Omega_{t}} v d x \quad \text { and } \quad \mathbb{E}_{\Omega_{t}}\left(\varphi_{i}\right)=0, \quad i=1,2,3,
$$

so

$$
\mathbb{E}_{\Omega_{t}}(u)=\mathbb{E}_{\Omega_{t}}(v)
$$

By Theorem 1 of [9] we have

$$
\partial_{x_{j}} w_{i}=\varepsilon_{i k l} \partial_{x_{k}} S_{j l}, \quad i=1,2,3, \quad w=\operatorname{rot} u,
$$

$S_{i j}=\partial_{x_{i}} u_{j}+\partial_{x_{j}} u_{i}$, so by (5.6) and Lemma 2.4 of [8] it follows that

$$
\|\operatorname{rot} u\|_{0, \Omega_{t}}^{2} \leq c \sum_{i, j=1}^{3}\left\|S_{i j}\right\|_{0, \Omega_{t}}^{2}=c \mathbb{E}_{\Omega_{t}}(u)=c \mathbb{E}_{\Omega_{t}}(v) .
$$

Employing the identity

$$
\partial_{x_{j}} u_{i}=\frac{1}{2}\left(\partial_{x_{j}} u_{i}+\partial_{x_{i}} u_{j}\right)+\frac{1}{2}\left(\partial_{x_{j}} u_{i}-\partial_{x_{i}} u_{j}\right)
$$

and (5.10) we have

$$
\|\nabla u\|_{0, \Omega_{t}}^{2} \leq c\left(\mathbb{E}_{\Omega_{t}}(u)+\|\operatorname{rot} u\|_{0, \Omega_{t}}^{2}\right) \leq c \mathbb{E}_{\Omega_{t}}(u)=c \mathbb{E}_{\Omega_{t}}(v)
$$

Using 5.3 we obtain

$$
\|\nabla v\|_{0, \Omega_{t}}^{2} \leq c\left(\mathbb{E}_{\Omega_{t}}(v)+|b|^{2}\right) .
$$

From Remark 3.2 using (5.3) we get systems of equations

$$
\sum_{i=1}^{3} b_{i} \int_{\Omega_{t}} \varphi_{i} \cdot \varphi_{j} d x=\int_{\Omega_{t}} u \varphi_{j} d x+\frac{1}{\varrho_{e}} \int_{\Omega_{t}}\left(\varrho-\varrho_{e}\right) v \cdot \varphi_{j} d x .
$$

Since det $\Gamma \neq 0$, where $\Gamma=\left\{\Gamma_{i j}\right\}, \Gamma_{i j}=\int_{\Omega_{t}} \varphi_{i} \varphi_{j} d x$ we can calculate $b$ from $(5.14)$, so

$$
|b|^{2} \leq c\left(\|u\|_{0, \Omega_{t}}^{2}+\left(\int_{\Omega_{t}}\left|\varrho-\varrho_{e}\right||v| d x\right)^{2}\right) .
$$


Now by Poincaré inequality and (5.7), (5.8), and Remark 3.1, we obtain

$$
\begin{aligned}
\|u\|_{0, \Omega_{t}}^{2} & \leq 2\left\|u-\frac{1}{\left|\Omega_{t}\right|} \int_{\Omega_{t}} u d x\right\|_{0, \Omega_{t}}^{2}+2\left\|\frac{1}{\left|\Omega_{t}\right|} \int_{\Omega_{t}} u d x\right\|_{0, \Omega_{t}}^{2} \leq \\
& \leq c\left(\|\nabla u\|_{0, \Omega_{t}}^{2}+\left\|\frac{1}{\left|\Omega_{t}\right|} \int_{\Omega_{t}} v d x\right\|_{0, \Omega_{t}}^{2}\right) \leq c\left(\mathbb{E}_{\Omega_{t}}(u)+\left(\int_{\Omega_{t}}\left|\varrho-\varrho_{e}\right||v| d x\right)^{2}\right) .
\end{aligned}
$$

From (5.3) and (5.14) we get

$$
\|v\|_{0, \Omega_{t}}^{2} \leq c\left(\|u\|_{0, \Omega_{t}}^{2}+\left(\int_{\Omega_{t}}\left|\varrho-\varrho_{e} \| v\right| d x\right)^{2}\right) .
$$

Then from (5.8), (5.12), (5.15) and (5.16) we get (5.2)

LEMMA 5.2. Let $\Omega_{t} \subset \mathbb{R}^{3}$ be a bounded domain. Let $\left(v, \varrho_{\sigma}\right)$ be a solution of $(1.1)_{1},(1.1)_{2}$, $(1.5)_{1}$ and $f=\int_{\Omega} v_{0} d x=\int_{\Omega} \varrho_{0} v_{0} \cdot \varphi_{i} d x, i=1,2,3$ and

$$
\mathbb{E}_{\Omega_{t}}\left(v_{t}\right)=\int_{\Omega_{t}}\left(\partial_{x_{i}} v_{j t}+\partial_{x_{j}} v_{i t}\right)^{2} d x<\infty
$$

Then there exists a constant $c>0$ such that

$$
\left\|v_{t}\right\|_{1, \Omega_{t}}^{2} \leq c\left(\mathbb{E}_{\Omega_{t}}\left(v_{t}\right)+\|v\|_{2, \Omega_{t}}^{4}+\left(\int_{\Omega_{t}}\left|\varrho-\varrho_{e}\right|\left|v_{t}\right| d x\right)^{2}\right) .
$$

Proof. Introduce a function $u$ by

$$
u=\sum_{i=1}^{3} b_{i} \varphi_{i}(x)+v_{t}
$$

where $\varphi_{i}=(x-\bar{x}) \times e_{i}, \quad \bar{x}=\frac{1}{\left|\Omega_{t}\right|}\left(\int_{\Omega_{t}} x_{1} d x, \int_{\Omega_{t}} x_{2} d x, \int_{\Omega_{t}} x_{3} d x\right), e_{i}=\left(\delta_{i 1}, \delta_{i 2}, \delta_{i 3}\right)$, $i=1,2,3$.

Define $b=\left(b_{1}, b_{2}, b_{3}\right)$ by

$$
b=\frac{1}{2\left|\Omega_{t}\right|} \int_{\Omega_{t}} \operatorname{rot} v_{t} d x .
$$

Since $\operatorname{rot} \varphi_{i}=2 e_{i}, i=1,2,3$, equations (5.19) and (5.20) imply

$$
\int_{\Omega_{t}} \operatorname{rot} u d x=0
$$

From (5.20) we have $\int_{\Omega_{t}} \varphi_{i} d x=0, i=1,2,3$ so

$$
\int_{\Omega_{t}} u d x=\int_{\Omega_{t}} v_{t} d x \quad \text { and } \quad \mathbb{E}_{\Omega_{t}}\left(\varphi_{i}\right)=0, \quad i=1,2,3,
$$

so

$$
\mathbb{E}_{\Omega_{t}}(u)=\mathbb{E}_{\Omega_{t}}\left(v_{t}\right)
$$

By Theorem 1 of [9] we have

$$
\partial_{x_{j}} w_{i}=\varepsilon_{i k l} \partial_{x_{k}} S_{j l}, \quad i=1,2,3, \quad w=\operatorname{rot} u,
$$


$S_{i j}=\partial_{x_{i}} u_{j}+\partial_{x_{j}} u_{i}$, so by (5.21) and Lemma 2.4 of [8] it follows that

$$
\|\operatorname{rot} u\|_{0, \Omega_{t}}^{2} \leq c \sum_{i, j=1}^{3}\left\|S_{i j}\right\|_{0, \Omega_{t}}^{2}=c \mathbb{E}_{\Omega_{t}}(u)=c \mathbb{E}_{\Omega_{t}}\left(v_{t}\right) .
$$

Employing the identity

$$
\partial_{x_{j}} u_{i}=\frac{1}{2}\left(\partial_{x_{j}} u_{i}+\partial_{x_{i}} u_{j}\right)+\frac{1}{2}\left(\partial_{x_{j}} u_{i}-\partial_{x_{i}} u_{j}\right)
$$

and (5.25) we have

$$
\|\nabla u\|_{0, \Omega_{t}}^{2} \leq c\left(\mathbb{E}_{\Omega_{t}}(u)+\|\operatorname{rot} u\|_{0, \Omega_{t}}^{2}\right) \leq c \mathbb{E}_{\Omega_{t}}(u)=c \mathbb{E}_{\Omega_{t}}\left(v_{t}\right) .
$$

Using (5.19) we obtain

$$
\left\|\nabla v_{t}\right\|_{0, \Omega_{t}}^{2} \leq c\left(\mathbb{E}_{\Omega_{t}}\left(v_{t}\right)+|b|^{2}\right)
$$

Integrating $(3.2)_{1}$ over $\Omega_{t}$ we get

$$
\int_{\Omega_{t}} v_{t} \varrho_{e} d x=-\int_{\Omega_{t}} \varrho v \cdot \nabla v d x-\int_{\Omega_{t}}\left(\varrho-\varrho_{e}\right) v_{t} d x
$$

and multiplying $(3.2)_{1}$ by $\varphi_{i}, i=1,2,3$ and integrating over $\Omega_{t}$ using (5.20) we get systems of equations

$$
\begin{aligned}
\sum_{j=1}^{3} b_{j} \int_{\Omega_{t}} \varrho_{e} \varphi_{i} \cdot \varphi_{j} d x= & \int_{\Omega_{t}} \varrho_{e} u \cdot \varphi_{i} d x+\int_{\Omega_{t}} \varrho v \cdot \nabla v \cdot \varphi_{i} d x \\
& +\int_{\Omega_{t}}\left(\varrho-\varrho_{e}\right) v_{t} \cdot \varphi_{i} d x .
\end{aligned}
$$

Since det $\Gamma \neq 0$, where $\Gamma=\left\{\Gamma_{i j}\right\}, \Gamma_{i j}=\int_{\Omega_{t}} \varphi_{i} \varphi_{j} d x$, we can calculate $b$ from (5.29), so

$$
|b|^{2} \leq c\left(\|u\|_{0, \Omega_{t}}^{2}+\|v\|_{2, \Omega_{t}}^{4}+\left(\int_{\Omega_{t}}\left|\varrho-\varrho_{e} \| v_{t}\right| d x\right)^{2}\right) .
$$

Now by Poincaré inequality and (5.22), (5.23), (5.28) we obtain

$$
\begin{aligned}
\|u\|_{0, \Omega_{t}}^{2} & \leq 2\left\|u-\frac{1}{\left|\Omega_{t}\right|} \int_{\Omega_{t}} u d x\right\|_{0, \Omega_{t}}^{2}+2\left\|\frac{1}{\left|\Omega_{t}\right|} \int u d x\right\|_{0, \Omega_{t}}^{2} \\
& \leq\left(\|\nabla u\|_{0, \Omega_{t}}^{2}+\left\|\frac{1}{\left|\Omega_{t}\right|} \int_{\Omega_{t}} v_{t} d x\right\|_{0, \Omega_{t}}^{2}\right) \\
& \leq c\left(\mathbb{E}_{\Omega_{t}}(u)+\|v\|_{2, \Omega_{t}}^{4}+\left(\int_{\Omega_{t}}\left|\varrho-\varrho_{e} \| v_{t}\right| d x\right)^{2}\right) .
\end{aligned}
$$

From (5.19) we get

$$
\left\|v_{t}\right\|_{0, \Omega_{t}}^{2} \leq c\left(\|u\|_{0, \Omega_{t}}^{2}+|b|^{2}\right)
$$

Then from (5.27), (5.30), (5.31) and (5.32) we get (5.18).

Similarly as Lemma 5.2 we prove 
Lemma 5.3. Let $\Omega_{t} \subset \mathbb{R}^{3}$ be a bounded domain. Let $\left(v, \varrho_{\sigma}\right)$ be a solution of $(1.1)_{1},(1.1)_{2}$, $(1.5)_{1}$ and $f=\int_{\Omega} v_{0} d x=\int_{\Omega} \varrho_{0} v_{o} \cdot \varphi_{i} d x, i=1,2,3$ and

$$
\mathbb{E}_{\Omega_{t}}\left(v_{t t}\right)=\int_{\Omega_{t}}\left(\partial_{x_{i}} v_{j t t}+\partial_{x_{j}} v_{i t t}\right)^{2} d x<\infty .
$$

Then there exists constant $c>0$ such that

$$
\begin{aligned}
\left\|v_{t t}\right\|_{1, \Omega_{t}}^{2} \leq & c\left(\mathbb{E}_{\Omega_{t}}\left(v_{t t}\right)+\left\|\varrho_{\sigma t}\right\|_{1, \Omega_{t}}^{2}\|v\|_{1, \Omega_{t}}^{4}\right. \\
& \left.+\|v\|_{1, \Omega_{t}}^{4}+\|v\|_{1, \Omega_{t}}^{2}\left\|v_{t}\right\|_{1, \Omega_{t}}^{2}+\left\|v_{t}\right\|_{0, \Omega_{t}}^{2}\left\|\varrho_{\sigma t}\right\|_{0, \Omega_{t}}^{2}+\left(\int_{\Omega_{t}}\left|\varrho-\varrho_{e}\right|\left|v_{t t}\right| d x\right)^{2}\right) .
\end{aligned}
$$

Acknowledgments. The author thanks Prof. W. Zajączkowski for very fruitful discussions during the preparation of this paper.

\section{References}

[1] S. Agmon, A. Douglis and L. Nirenberg, Estimates near the boundary for solutions of elliptic partial differential equations satisfying general boundary conditions, I, II Comm. Pure Appl. Math. 12 (1959), 623-727, 17 (1964), 35-92.

[2] O. V. Besov, V. P. Il'in and S. M. Nikolskii, Integral Representations of Functions and Imbedding Theorems, Nauka, Moscow, 1975.

[3] L. Landau and E. Lifschitz, Electrodynamics of Continuum Media, Nauka, Moscow, 1957.

[4] P. Kacprzyk, Local existence of solutions of the free boundary problem for the equations of magnetohydrodynamic incompresible fluid, Appl. Math. 30(2003), 461-488.

[5] P. Kacprzyk, Almost global existence of solutions of the free boundary problem for the equations of magnetohydrodynamic incompresible fluid, Appl. Math. 31 (2004), 69-77.

[6] P. Kacprzyk, Local existence of solutions of the free boundary problem for the equations of magnetohydrodynamic compresible fluid, Appl. Math. 31 (2004), 209-227.

[7] P. Kacprzyk, Global existence of solutions of the free boundary problem for the equations of magnetohydrodynamic compresible fluid, TMNA 23 (2004), 339-356.

[8] O. Ladyzhenskaya and V. Solonnikow, On some problems of vector analysis and generalized formulations on boundary problems for Navier-Stokes equations, Zap. Nauchn. Sem. LOMI 59 (1976).

[9] V. Solonnikov, On an unisteady motion of an isolated volume of a viscous incompresible fluid, Izv. Akad. Nauk SSSR Ser. Mat 51 (1987).

[10] V. A. Solonnikov and A. Tani, Evolution free boundary problem for equations of motion of viscous compressible barotropic liquid, prepr. Paderborn Univ.

[11] G. Ströhmer and W. Zajączkowski, Local existence of solutions of the free boundary problem for the equations of compressible barotropic viscous self-gravitating fluids, Appl. Math. 26 (1999), 1-31.

[12] G. Ströhmer and W. M. Zajączkowski, On the existence and properties of rotationally symmetric equilibrium states of compressible barotropic self-gravitating fluids, Indiana Univ. Math. J. 46 (1997), 1181-1220.

[13] E. Zadrzyńska, On nonstationary motion of a fixed mass of a general viscous compressible heat conducting capilary fluid bounded by a free boundary, Appl. Math. 25 (1999), 489-511. 
[14] E. Zadrzyńska, Free boundary problem for a viscous heat-conducting flow with surface tension, Topol. Methods Nonlin. Anal. 19 (2002).

[15] E. Zadrzyńska and W. Zajączkowski, On local motion of a general compressible viscous heat conducting fluid bounded by a free surface, Ann. Polon. Math. 59 (1994), 133-170.

[16] E. Zadrzyńska and W. M. Zajączkowski, On the global existence theorem for a free boundary problem for equations of a viscous compresible heat conducting fluid, Ann. Polon. Math. 63 (1996), 199-221.

[17] E. Zadrzyńska and W. Zajączkowski, On the global existence theorem for a free boundary problem for equations of a viscous compressible heat conducting capillary fluid, J. Appl. Anal. 2 (1996), 125-169.

[18] E. Zadrzyńska and W. Zajączkowski, Local existence of solutions of a free boundary problem for equations of compressible visocus heat-conducting fluids, Appl. Math. 25 (1998), 179-220.

[19] W. M. Zajączkowski, Existence of local solutions for free boundary problems for viscous compressible barotropic fluids, Ann. Polon. Math. 60 (1995), 255-287.

[20] W. M. Zajączkowski, On nonstationary motion of a compresible barotropic viscous fluid bounded by a free surface, Dissertationes Math. 324 (1993).

[21] W. M. Zajączkowski, On nonstationary motion of a compresible barotropic viscous capillary fluid bounded by a free surface, SIAM J. Math. Anal. 25 (1994), 1-84. 